\title{
Goodwillie's Calculus of Functors and Higher Topos Theory
}

\author{
Mathieu Anel*, Georg Biedermann ${ }^{\dagger}, \quad$ Eric Finster ${ }^{\ddagger}$, \\ and André Joyal§
}

\begin{abstract}
We develop an approach to Goodwillie's Calculus of Functors using the techniques of higher topos theory. Central to our method is the introduction of the notion of fiberwise orthogonality, a strengthening of ordinary orthogonality which allows us to give a number of useful characterizations of the class of $n$-excisive maps. We use these results to show that the pushout product of a $P_{n}$-equivalence with a $P_{m}$-equivalence is a $P_{m+n+1}$ equivalence. Then, building on our previous work [ABFJ17, we prove a Blakers-Massey type theorem for the Goodwillie tower of functors. We show how to use the resulting techniques to rederive some foundational theorems in the subject, such as delooping of homogeneous functors.
\end{abstract}

\section{Contents}

1 Introduction 2

2 Prerequisites 5

2.1 Topoi . . . . . . . . . . . . . . . . . 5

2.2 Cubes, gaps and cogaps . . . . . . . . . . . . . . . 6

2.3 Pushout product and pullback hom . . . . . . . . . . . . 7

2.4 Orthogonality conditions . . . . . . . . . . . . . . 10

2.5 The fiberwise diagonal map . . . . . . . . . . . . 14

2.6 Modalities and generalized Blakers-Massey theorems . . . . . . 17

3 The Goodwillie Localization $\quad 19$

3.1 The $n$-excisive modality . . . . . . . . . . . . . . 20

3.2 Cubical Diagrams and Orthogonality . . . . . . . . . . . 22

3.3 A Characterization of $n$-excisive maps . . . . . . . . . 28

*SPHERE, UMR 7219, Univ. Paris Diderot. mathieu.anel@gmail.com

${ }^{\dagger}$ LAGA (UMR7539), Institut Galilée, Univ. Paris 13; new address: Universidad del Norte, Departamento de Matemáticas y Estadística, Barranquilla, Colombia, gbm@posteo.de

‡IRIF, Univ. Paris Diderot. ericfinster@gmail.com

§CIRGET, UQÀM. joyal.andre@uqam.ca 
3.4 The Blakers-Massey Theorem for the Goodwillie Tower . . . . . . 31

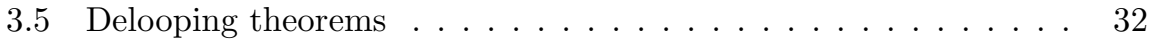

A Truncated and connected maps of $n$-excisive functors 35

\section{Introduction}

Goodwillie's calculus of homotopy functors Goo90, Goo92, Goo03 is a powerful technique in homotopy theory for approximating possibly complicated functors by simpler ones using a generalized notion of excision. In particular, applied to the identity functor on the category of spaces, it produces a filtration interpolating between stable and unstable homotopy which has proved extremely useful in calculations.

In this article, we revisit some of the foundations of the subject from the point of view of higher topos theory. In particular, we will show that many of the fundamental results can be deduced from the following Blakers-Massey type theorem, which we feel is of independent interest.

Theorem 3.4.1 Let

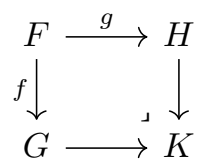

be a homotopy pushout square of functors. If $f$ is a $P_{m}$-equivalence and $g$ is a $P_{n}$-equivalence, then the induced cartesian gap map

$$
(f, g): F \rightarrow G \times_{K} H
$$

is a $P_{m+n+1}$-equivalence.

The second result is a "dual" version.

Theorem 3.4.2 Let

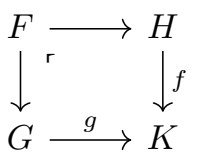

be a homotopy pullback square of functors. If $f$ is a $P_{m}$-equivalence and $g$ is a $P_{n}$-equivalence, then the cocartesian gap map

$$
\lfloor f, g\rfloor: G \sqcup_{F} H \rightarrow K
$$

is a $P_{m+n+1}$-equivalence.

We show how to rederive known delooping results in homotopy functor calculus in an easy and conceptual way as consequences. In particular, we obtain a new proof of Goodwillie's Lemma 2.2 Goo03 that homogeneous functors deloop, independent of the material of [Goo03, Section 2] 
Both of these results rest on the material of the companion article [ABFJ17] where a very general version of the Blakers-Massey theorem was proved. There, the language of higher topoi was adopted, and we find it equally well suited for the calculus of homotopy functors, particularly because $n$-excisive functors to spaces form a higher topos. Indeed many of the results of this article arise from working systematically fiberwise, a method very much encouraged by the topostheoretic point of view. Given this general framework, it will thus be convenient to drop any reference to higher derived structures and take them for granted. When we talk about a "category", we mean " $\infty$-category" and all (co-)limits are to be interpreted as $\infty$-categorical (co-)limits. In particular, we will not use the terms "homotopy (co-)limit", as was done above for the sake of introduction. The $\infty$-categorical machinery already describes a derived, homotopy invariant setting with all higher coherences. We will fequently say "isomorphism" for what is perhaps more commonly called "weak equivalence". Similarly, mapping spaces or internal hom objects are always to be taken derived. The reader who finds this article easier to read by using model structures should not have any difficulties in doing so.

The main tool of our paper ABFJ17. was the notion of modality, and it will be equally important here. A modality is a unique factorization system whose left and right class are closed under base change. An example is the factorization of a map of spaces into an $n$-connected map followed by an $n$ truncated map. Application of our generalized Blakers-Massey theorem to this example leads to the classical version of the theorem. Here we observe that the factorization of a natural transformation into a $P_{n}$-equivalence followed by an $n$-excisive map is a modality in a presheaf topos. That this is the case is a consequence of the fact that Goodwillie's $n$-excisive approximation $P_{n}$ is, in fact, a left exact localization of the topos of functors. The left classes of these $n$-excisive modalities for various $n \geq 0$ are compatible with the pushout product in the following sense:

Theorem 3.3.4) (4) The pushout product of a $P_{m}$-equivalence with a $P_{n}$-equivalence is a $P_{m+n+1}$-equivalence.

This fact immediately implies the main theorems by means of the generalized Blakers-Massey theorems from [ABFJ17]. It also yields that the smash or join of an $m$-reduced functor with an $n$-reduced functor is $(m+n-1)$-reduced, see Example 3.3.5. In order to prove this theorem we need to take a step back and develop systematically a fiberwise approach, which is to say, concentrate our attention on constructions which are compatible with base change. Indeed, one characterization of a modality is a factorization system whose left and right classes determine each other via fiberwise orthogonality, a notion which we introduce in Definition 2.4.6. Briefly, two maps are fiberwise orthogonal if all of their base changes are externally orthogonal to each other. As is the case in the theory of ordinary factorization systems, this condition can be reformulated as say that a certain map, which we term the fiberwise diagonal (Definition 2.5.1) is an isomorphism. The resulting adjunction tricks exploited in Proposition 2.5.4 lead us to Theorem 3.3.1 where we prove that the $n$-excisive modalities are 
generated by pushout product powers of certain explicit generating maps.

From here, Theorems 3.4.1 and 3.4.2 which provide analogues of the BlakersMassey theorems for the Goodwillie tower are easily deduced. This allows us in Theorem 3.5 .2 to find a classifying map for the map $P_{n} F \rightarrow P_{n-1} F$ in the Goodwillie tower and reprove delooping results (Corollaries 3.5.3 and 3.5.5) for functors whose derivatives live only in a certain range; in particular homogeneous functors are infinitely deloopable.

To justify a portion of the result in 3.5.2, and because we feel it is of independent interest, Appendix $\mathrm{A}$ is included. In Theorem A.0.5 we give a characterization of monomorphisms and covers (effective epimorphisms) in the topos $[\mathcal{C}, \mathcal{S}]^{(n)}$ of $n$-excisive functors. As far as we know this is the first time $n$-excisive functors are studied in detail as a topos and we wish to advertise this as a fruitful line of thought.

Finally, a few remarks are in order about the overall placement of our results in the larger landscape of studies of the Goodwillie Calculus. Indeed, by now there are many versions of Goodwillie-style filtrations which appear in a number of different contexts. What is traditionally known as the homotopy calculus and concerns excisive properties of functors (defined by their behavior on certain cubical diagrams) can be developed in a very general setting as is done, for example, model category theoretically in [Kuh07] or $\infty$-categorically in Lur16. From the point of view of this theory, the results of this article are somewhat restricted: our arguments require that the functors under study have as codomain an $\infty$-topos. In particular, this means we can not immediately apply our results to functors taking values in stable categories, such as, for example, spectra. It remains for future work to understand to what extent our techniques might be applied to the stable case.

From another point of view, however, our results can be seen as providing a generalization of the homotopy calculus. For example, when we restrict to functors with values in a topos, our Theorem 3.3.1 leads to a completely internal characterization of the construction of the Goodwillie tower which makes no mention of cubical diagrams. In upcoming work we will show how these techniques can be applied to give a uniform treatment of other varieties of Goodwillie calculus such as the orthogonal calculus Wei95 where the approximation scheme is not necessarily defined in terms of the behavior of a functor with respect to limits and colimits.

This last point perhaps best illustrates the philosophy of our approach. Indeed, while the theory of higher topoi has applications to Goodwillie calculus, providing streamlined and conceptual proofs of its main results, the reverse is also true: we can use the Goodwillie calculus as tool in the study of higher topoi themselves. We feel that much remains to be done in developing this point of view.

Acknowledgment: The first author has received funding from the European Research Council under the European Community's Seventh Framework Programme (FP7/2007-2013 Grant Agreement n²63523). The second author and this project have received funding from the European Unions Hori- 
zon 2020 research and innovation programme under Marie Skłodowska-Curie grant agreement No 661067. The second author also acknowledges support from the project ANR-16-CE40-0003 ChroK. The third author has been supported by the CoqHoTT ERC Grant 64399. The fourth author has been supported by the NSERCC grant 371436.

\section{Prerequisites}

In this section we recall material from the our companion paper ABFJ17. In particular, we give the definition of a modality 2.6.1] and state our generalized Blakers-Massey theorems 2.6.7 and 2.6.8.

\subsection{Topoi}

This article is written using the language of higher topoi. For an outline of the theory we refer the reader to [Rez05, Joy08, Lur09]. A very brief overview of the essential properties tailored to our needs is given in [ABFJ17, Section 2]. We will now drop $\infty$ from the notation and refer to them simply as topoi. We write $\mathcal{S}$ for the category of spaces. We will denote the category of space-valued functors $\mathcal{C} \rightarrow \mathcal{S}$ on a small category $\mathcal{C}$ by $[\mathcal{C}, \mathcal{S}]$. A functor $\mathcal{C} \rightarrow \mathcal{S}$ is a presheaf on $\mathcal{C}^{\text {op }}$.

Definition 2.1.1. A topos is an accessible left exact localization of a presheaf category $[\mathcal{C}, \mathcal{S}]$ for some small category $\mathcal{C}$.

The reader should be aware that "left exact localization" is to be taken in the derived sense. Spelled out in the language of model categories it means "left Bousfield localization commuting with finite homotopy limits up to weak equivalence". This is in line with the general approach in this article that everything should be interpreted in $\infty$-categorical terms. We recall that when we speak of (co-)limits, the corresponding notions in the language of model structures are homotopy (co-)limits.

Example 2.1.2. The primary examples of topoi of interest to us here are:

1. The category $\mathcal{S}$ of spaces (as modelled by topological spaces or simplicial sets with weak homotopy equivalences) is the prime example of a topos.

2. The category $[\mathcal{C}, \mathcal{S}]$ of functors to spaces is a topos.

3. The full subcategory $[\mathcal{C}, \mathcal{S}]^{(n)} \subset[\mathcal{C}, \mathcal{S}]$ of $n$-excisive functors, which, as explained in Example 2.6.5, is itself a topos.

We recall that within a topos colimits are preserved by base change. 


\subsection{Cubes, gaps and cogaps}

Let $\underline{n}=\{1, \cdots, n\}$ and write $P(\underline{n})$ for the poset of its subsets. Define $P_{0}(\underline{n})$ to be the poset of non-empty subsets; let $P_{1}(\underline{n})$ be the poset of proper subsets.

Now consider a finitely complete category $\mathcal{E}$. An $n$-cube in a category $\mathcal{E}$ is a functor $X: P(\underline{n}) \rightarrow \mathcal{E}$. We will refer to the canonical map

$$
X(\varnothing) \rightarrow \lim _{U \in P_{0}(\underline{n})} x(U)
$$

as the cartesian gap map or simply the gap map for brevity. An $n$-cube is said to be cartesian if its gap map is an isomorphism. For example, a 2-cube is cartesian if and only if it is a pullback square.

For an $n$-cube $\mathcal{X}$ in a finitely cocomplete category $\mathcal{E}$ there also exists the canonical map

$$
\underset{U \in P(\underline{n})}{\operatorname{colim}} X(U) \rightarrow X(\underline{n})
$$

which we will call it the cocartesian gap map or briefly, cogap map. An $n$-cube is cocartesian if its cogap map is an isomorphism. A square is cocartesian if and only if it is a pushout square.

An $n$-cube is called strongly cartesian (resp. strongly cocartesian) if all its 2-dimensional subcubes are cartesian (resp. cocartesian).

Definition 2.2.1. The external cartesian product of two cubical diagrams $X$ : $P(\underline{m}) \rightarrow \mathcal{E}$ and $\mathcal{y}: P(\underline{n}) \rightarrow \mathcal{E}$ is a cubical diagram $X \otimes y: P(\underline{m+n})=P(\underline{m}) \times$ $P(\underline{n}) \rightarrow \mathcal{E}$ defined by putting

$$
\left(X_{\square} y\right)(A, B)=X(A) \times y(B)
$$

for every $A \in P(\underline{m})$ and $B \in P(\underline{n})$. The external coproduct $X_{\boxplus} y: P(\underline{m+n})=$ $P(\underline{m}) \times P(\underline{n}) \rightarrow \mathcal{E}$ is defined by putting

$$
(X \boxplus y)(A, B)=X(A) \sqcup y(B) .
$$

The external cartesian (co)product of two strongly (co)cartesian cubes is strongly (co)cartesian. Every map $f: A \rightarrow B$ defines a 1-cube $f: P(\underline{1}) \rightarrow \mathcal{E}$. The $n$-cube

$$
f_{1} \otimes \cdots \otimes f_{n}: P(\underline{n}) \rightarrow \mathcal{E}
$$

is strongly cartesian and the $n$-cube

$$
f_{1} \boxplus \cdots \boxplus f_{n}: P(\underline{n}) \rightarrow \mathcal{E}
$$

is strongly cocartesian for any sequence of maps $\left\{f_{i}: K_{i} \rightarrow L_{i}\right\}_{i=1}^{n}$. In particular, the square

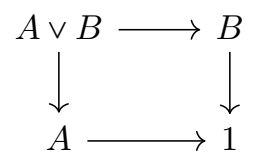


is cocartesian for any pair of objects in a pointed category.

Now let $\mathcal{E}$ be finitely complete and finitely cocomplete. Given a commutative square in $\mathcal{E}$ :

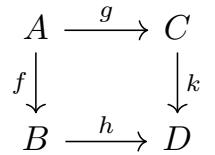

We will denote the gap map by

$$
(f, g): A \rightarrow B \times{ }_{D} C .
$$

The cogap map of the square will be denoted by

$$
\lfloor h, k\rfloor: B \cup_{A} C \rightarrow D .
$$

Strictly speaking these maps depend on the whole square. In practice the remaining maps will always be clear from the context.

\subsection{Pushout product and pullback hom}

Let $\mathcal{E}$ be a topos. For any two maps $u: A \rightarrow B$ and $v: S \rightarrow T$ in $\mathcal{E}$ the square

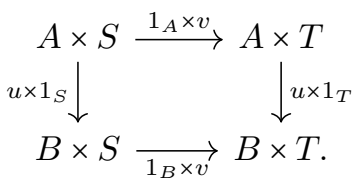

is cartesian, and we define the pushout product of $u$ and $v$, denoted $u \square v$, to be the cocartesian gap map of the previous square:

$$
u \square v=A \times T \sqcup_{A \times S} B \times S \rightarrow B \times T .
$$

Let 0 and 1 be respectively an initial and a terminal object for $\mathcal{E}$. A topos $\mathcal{E}$ has a strict initial object which means that any arrow $C \rightarrow 0$ is an isomorphism. In particular, since $\mathcal{E}$ has finite products, this implies $0 \times X=0$ for all objects $X \in \mathcal{E}$. The pushout product defines a symmetric monoidal structure on the category $\mathcal{E}^{\rightarrow}$ of arrows, with unit $0 \rightarrow 1$. In particular, we have $u \square v=v \square u$ and $(u \square v) \square w=u \square(v \square w)$.

Example 2.3.1. We give some examples of pushout products that will be useful in the sequel.

1. The iterated pushout product $f_{1} \square \cdots \square f_{n}$ of a sequence of maps $\left\{f_{i}: K_{i} \rightarrow\right.$ $\left.L_{i}\right\}_{i=1}^{n}$ is the cogap map of $n$-cube $f_{1} \otimes \cdots \otimes f_{n}$.

2. For any map $A \rightarrow B$ in $\mathcal{E}$ and any object $C$, the map $(0 \rightarrow C) \square(A \rightarrow B)$ is simply

$$
(0 \rightarrow C) \square(A \rightarrow B)=C \times A \rightarrow C \times B
$$


3. For two pointed objects $1 \rightarrow A$ and $1 \rightarrow B$ in $\mathcal{E}$, we have

$$
(1 \rightarrow A) \square(1 \rightarrow B)=A \vee B \rightarrow A \times B
$$

the canonical inclusion of the wedge into the product.

4. Recall that the join of two objects $A$ and $B$ in $\mathcal{E}$, denoted $A \star B$, is the pushout of the diagram $A \leftarrow A \times B \rightarrow B$. One sees immediately that

$$
(A \rightarrow 1) \square(B \rightarrow 1)=A \star B \rightarrow 1 .
$$

5. The fiberwise join $X \star_{B} Y$ of two maps $f: X \rightarrow B$ and $g: Y \rightarrow B$ is the pushout of the diagram $X \leftarrow X \times_{B} Y \rightarrow Y$. It is the pullback of the map $f \square g$ along the diagonal $B \rightarrow B \times B$

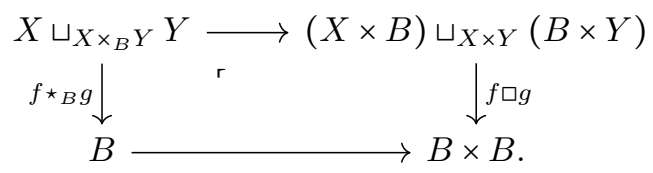

The name fiberwise join is justified by the fact that for $b \in B$, we have the identification

$$
\operatorname{fib}_{b}\left(X \star_{B} Y\right)=\left(\operatorname{fib}_{b} f\right) \star\left(\operatorname{fib}_{b} g\right)
$$

since colimits are stable by base change in the topos. More generally, the iterated fiberwise join $X_{1} \star_{B} \cdots \star_{B} X_{n}$ of a sequence of maps $f_{i}: X_{i} \rightarrow B$ $(1 \leq i \leq n)$ with codomain $B$ is the the pullback of the map $f_{1} \square \cdots \square f_{n}$ along the diagonal $B \rightarrow B^{n}$.

6. Since colimits in $\mathcal{E}$ commute with base change, the pushout product $f \square g$ can be thought as the "external" join product of the fibers of $f$ and $g$. An easy computation shows that the fiber of the map $(f: A \rightarrow B) \square(g: C \rightarrow D)$ at a point $(b, d) \in B \times D$ is the join of the fibers of $f$ and $g$. Details can be found in ABFJ17, Rem. 2.4].

7. For an object $Z$ the slice category $\varepsilon / Z$ has its own pushout product denoted $\square_{Z}$. Given $f: A \rightarrow B$ and $g: X \rightarrow Y$ in $\mathcal{E} / Z$, the corresponding formula reads

$$
f \square_{Z} g=\left(A \times_{Z} Y\right) \cup_{\left(A \times_{Z} X\right)}\left(B \times_{Z} X\right) \rightarrow\left(B \times_{Z} Y\right) .
$$

We will make use of these observations in Section 3 in order to relate the calculus of strongly cocartesian diagrams in a category $\mathcal{C}$ with that of orthogonality in the presheaf category $[\mathcal{C}, \mathcal{S}]$.

For two objects $A, B$ of $\mathcal{E}$, we let $[A, B]$ be the space of maps from $A$ to $B$ in $\mathcal{E}$. For two maps $u: A \rightarrow B$ and $f: X \rightarrow Y$ in $\mathcal{E}$ we consider the following 
commutative square in $\mathcal{S}$

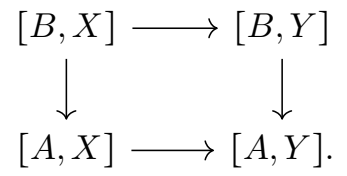

We define the external pullback hom $\langle u, f\rangle$ to be the cartesian gap map of the previous square:

$$
\langle u, f\rangle:[B, X] \rightarrow[A, X] \times[A, Y][B, Y] .
$$

Let $\llbracket A, B \rrbracket$ denote the internal hom object in $\mathcal{E}$. Then we can define similarly an internal pullback hom

$$
\langle u, f\rangle: \llbracket B, X \rrbracket \rightarrow \llbracket A, X \rrbracket \times_{\llbracket A, Y \rrbracket} \llbracket B, Y \rrbracket,
$$

which is a map in $\mathcal{E}$.

Example 2.3.2. The internal pullback hom has a number of useful special cases:

1. The category of spaces $\mathcal{S}$ is cartesian closed and we have $\langle\langle u, f\rangle=\langle u, f\rangle$ for any pair of maps $u, f \in \mathcal{S}$.

2. If $S^{0}=1 \sqcup 1$ is the 0 -sphere in $\mathcal{E}$, then

$$
\left\langle S^{0} \rightarrow 1, X \rightarrow 1\right\rangle=X \rightarrow X \times X
$$

is the diagonal map of $X$. Similarly, for any map $f: X \rightarrow Y$ the map

$$
\left\langle\left\langle S^{0} \rightarrow 1, f\right\rangle=X \rightarrow X \times_{Y} X\right.
$$

is the diagonal map $\Delta f$ of $f$.

3. More generally, for any object $A$ in $\mathcal{E}$, the map

$$
\Delta_{A}(X)=\langle\langle A \rightarrow 1, X \rightarrow 1\rangle: X \rightarrow \llbracket A, X \rrbracket
$$

is the $A$-diagonal of $X$. Similarly, for any map $f: X \rightarrow Y$ the map $\langle\langle A \rightarrow 1, f\rangle$ defines the $A$-diagonal of $f$.

It is useful to keep in mind that the global section functor $\Gamma:=[1,-]: \mathcal{E} \rightarrow \mathcal{S}$ takes the object $\llbracket A, B \rrbracket$ to the space $[A, B]$ :

$$
\Gamma(\llbracket A, B \rrbracket)=[1, \llbracket A, B \rrbracket]=[A, B] .
$$

Since $\Gamma$ commutes with all limits, one has

$$
\Gamma(\langle f f, g\rangle)=[1,\langle\langle f, g\rangle]=\langle f, g\rangle .
$$


For any $Z \in \mathcal{E}$, the slice topos $\mathcal{E}_{/ Z}$ has an internal hom and an internal pullback hom that we will denote respectively by $\llbracket-,-\rrbracket_{Z}$ and $\left\langle\langle-,-\rangle_{Z}\right.$. The base change $u^{*}: \mathcal{E}_{/ Z} \rightarrow \mathcal{E}_{/ T}$ along a map $u: T \rightarrow Z$, preserves cartesian products and internal homs. For two objects $A, B$ in $\mathcal{E}_{/ Z}$, we have a canonical isomorphism

$$
u^{*} \llbracket A, B \rrbracket_{Z}=\llbracket u^{*} A, u^{*} B \rrbracket_{T} .
$$

We leave to the reader the proof of the following lemma asserting that the same formula is true for the internal pullback hom.

Lemma 2.3.3. For two maps $f: A \rightarrow B$ and $g: X \rightarrow Y$ in $\mathcal{E}_{/ Z}$, and any map $u: T \rightarrow Z$ we have a canonical isomorphism in $\mathcal{E}_{/ T}$ :

$$
u^{*}\left\langle\langle f, g\rangle_{Z}=\left\langle\left\langle u^{*} f, u^{*} g\right\rangle_{T} .\right.\right.
$$

Remark 2.3.4. The external and internal pullback hom define functors

$$
\left.\langle-,-\rangle:\left(\mathcal{E}^{\rightarrow}\right)^{\mathrm{op}} \times \mathcal{E}^{\rightarrow} \rightarrow \mathcal{S}^{\rightarrow} \quad \text { and } \quad\langle-,-\rangle\right\rangle:\left(\mathcal{E}^{\rightarrow}\right)^{\mathrm{op}} \times \mathcal{E}^{\rightarrow} \rightarrow \mathcal{E}^{\rightarrow} .
$$

Together with the pushout product the internal pullback hom yields a closed symmetric monoidal structure on $\mathcal{E} \rightarrow$. In particular, we have

$$
\langle\langle f \square g, h\rangle=\langle\langle f,\langle\langle g, h\rangle\rangle\rangle .
$$

We have also the relation $\langle f \square g, h\rangle=\langle f,\langle\langle g, h\rangle\rangle\rangle$.

\subsection{Orthogonality conditions}

In this section, we define and compare three notions of orthogonality for maps: the external orthogonality $\perp$ and internal orthogonality $\perp$, which will be related to the internal and external pullback hom, and the new fiberwise orthogonality $\Perp$ (Definition 2.4.6), which will be related to a variation of the pullback hom in Section 2.5]

Although our focus is mainly on $\underline{\Perp}$, it is convenient to formulate its properties as properties of $\perp$. So we provide some recollection on the matter. The relation $\perp$ is introduced only for comparison purposes and to avoid any confusion between $\underline{\Perp}$ and $\Perp$.

Let us point out that our motivation for introducing fiberwise orthogonality and the fiberwise diagonal is to prove Proposition 2.5.4. This eventually leads to Theorem 3.3.4 that is our new ingredient to Goodwillie calculus that lets us prove the Blakers-Massey Theorem for the Goodwillie tower.

Definition 2.4.1. Two maps $f: A \rightarrow B$ and $g: X \rightarrow Y$ in $\mathcal{E}$ are externally orthogonal or simply orthogonal if the map $\langle f, g\rangle$ is an isomorphism in $\mathcal{S}$. We write $f \perp g$ for this relation and we say that $f$ is externally left orthogonal to $g$ and that $g$ is externally right orthogonal to $f$. 
Unfolding the definitions, one immediately verifies that if $f \perp g$ then for every commutative square

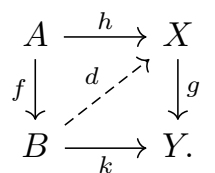

the space of diagonal fillers is contractible, that is to say, a digonal filler exists and is unique up to homotopy.

Recall that for a topos $\mathcal{E}$, all slice categories $\mathcal{E}_{/ Z}$ are also topoi. Therefore, each $\varepsilon_{/ Z}$ has an external orthogonality relation which we will denote by $\perp_{Z}$.

Definition 2.4.2. We will say that two maps $f: A \rightarrow B$ and $g: X \rightarrow Y$ are internally orthogonal, and write $f \pm g$, if the map $\langle\langle f, g\rangle$ is an isomorphism in $\mathcal{E}$. Similarly we say that $f$ is internally left orthogonal to $g$ and that $g$ is internally right orthogonal to $f$.

For an object $Z$ and a map $f: A \rightarrow B$ we write $Z \times f: Z \times A \stackrel{\operatorname{id}_{Z} \times f}{\longrightarrow} Z \times B$. We leave to the reader the proof of the following lemma.

Lemma 2.4.3. The following conditions are equivalent:

(1) $f \pm g$

(2) For any $Z \in \mathcal{E}$ we have $(Z \times f) \perp g$.

In particular, $f \perp g$ implies $f \perp g$.

Since each slice topos $\varepsilon_{/ Z}$ has its own internal hom objects it has an internal orthogonality relation which we will denote by $\Perp_{Z}$. The following lemma proves that these internal orthogonality relations are compatible with base change.

Lemma 2.4.4. For any two maps $f: A \rightarrow B$ and $g: X \rightarrow Y$ in $\mathcal{E}$, and for any object $Z \in \mathcal{E}$ we have

$$
f \pm g \quad \Longrightarrow \quad Z^{*} f \Perp_{Z} Z^{*} g
$$

where $Z^{*}$ is the base change functor along the map $Z \rightarrow 1$. Moreover, the converse is true if the map $Z \rightarrow 1$ is a cover.

Proof. If $u: Z \rightarrow 1$, then by Lemma 2.3.3, we have a canonical isomorphism

$$
u^{*}\left\langle\langle f, g\rangle=\left\langle\left\langle u^{*} f, u^{*} g\right\rangle_{Z} .\right.\right.
$$

This proves that $f \pm g \Rightarrow u^{*} f \Perp_{Z} u^{*} g$. The converse is true since the functor $u^{*}$ is conservative when $u$ is a cover.

The following proposition lists several equivalent properties that will be used to define fiberwise orthogonality. In order to facilitate the reading, we employ the following convention in the proofs which follow: given a map $f: A \rightarrow B$ and a map $u: Z \rightarrow B$, we denote by $f_{Z}$ the map $u^{*} f: A \times_{B} Z \rightarrow Z$. The point of this notation is to make $u$ implicit, remembering only the new base. The context will make clear along which map the base is changed. 
Proposition 2.4.5. Given two maps $f: A \rightarrow B$ and $g: X \rightarrow Y$ in $\mathcal{E}$, the following conditions are equivalent:

(1) For any $Z \in \mathcal{E}$ and any maps $b: Z \rightarrow B$ and $y: Z \rightarrow Y$, it is true in $\mathcal{E}_{/ Z}$ that

$$
f_{Z} \doteq Z g_{Z}
$$

(2) The base changes of $f$ and $g$ onto $B \times Y$ along the projections to $B$ and $Y$ satisfy

$$
f_{B \times Y} \stackrel{\perp}{B \times Y} g_{B \times Y} .
$$

(3) The diagonal map in $\mathcal{E}_{/ B \times Y}$

$$
\Delta_{f_{B \times Y}}\left(g_{B \times Y}\right): g_{B \times Y} \rightarrow \llbracket f_{B \times Y}, g_{B \times Y} \rrbracket_{B \times Y}
$$

is an isomorphism (see Example 2.3.213).

(4) For any $Z \rightarrow B \times Y$ and any $T \rightarrow Z$ we have

$$
f_{T} \perp_{Z} g_{Z}
$$

(5) For any $Z \in \mathcal{E}$ and any maps $b: Z \rightarrow B$ and $y: Z \rightarrow Y$, it is true in $\mathcal{E}_{/ Z}$ that

$$
f_{Z} \perp_{Z} g_{Z}
$$

(6) For any two maps $Z \rightarrow B$ and $Z^{\prime} \rightarrow Y$ we have $f_{Z} \perp g_{Z^{\prime}}$.

(7) For any map $Z \rightarrow B$ we have $f_{Z} \perp g$.

Proof. (1) $\Rightarrow(2)$ This is obvious since (2) is a special case of (1).

$(2) \Rightarrow(1)$ This follows from Lemma 2.4.4 that states that orthogonality is stable by base change.

$(2) \Leftrightarrow(3)$ This is equivalent by the definition of orthogonality in $\mathcal{E}_{B \times Y}$.

$(1) \Leftrightarrow(4)$ This is Lemma 2.4 .3 applied to the topos $\mathcal{E}_{/ Z}$.

(4) $\Rightarrow(5)$ Set $T \rightarrow Z=\mathrm{id}_{Z}$.

(5) $\Leftrightarrow(6)$ We need to prove that for all $Z$ and all $B \leftarrow Z \rightarrow Y$,

$$
f_{Z} \perp_{Z} g_{Z} \Longleftrightarrow \forall U \rightarrow B, \forall T \rightarrow Y, f_{U} \perp g_{T} .
$$

We consider the following diagram

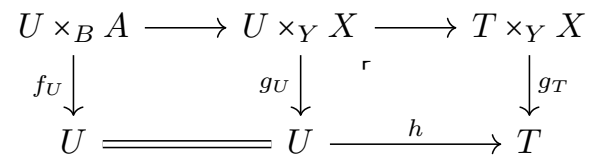

where $h$ is arbitrary and the right square is cartesian. Because the right square is cartesian, the space of diagonal fillers of the outer square is equivalent to that of the left square. When $h$ varies, the former condition gives $f_{U} \perp g_{T}$ and the latter $f_{U} \perp_{U} g_{U}$, hence proving their equivalence. 
$(6) \Leftrightarrow(7)$ Since it is clear that $(6) \Rightarrow(7)$, we need to show the other implication. Let $f_{U}$ be the base change of $f$ along some map $U \rightarrow B$, and $g_{T}$ the base change of $g$ along some map $h: T \rightarrow Y$, we consider the following diagram where the left square is commutative and the right square is cartesian

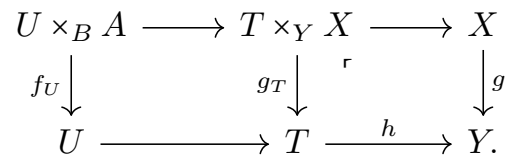

Again, because the right square is cartesian, the space of diagonal fillers of the outer square is equivalent to that of the left square, which proves $(7) \Rightarrow(6)$.

$(7) \Rightarrow(4)$ Let us consider the following diagram

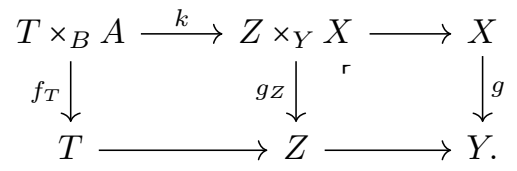

where the right square is cartesian and $k$ is any map such that the left square is commutative. Condition (4) says that for any such $k$ the space of fillers of the left square is contractible. Since the right square is cartesian this is equivalent to the outer square having a contractible space of fillers. But Condition (7) states that any map from $f_{T}$ to $g$, i.e. a commutative square, has a contractible space of fillers. So (7) implies (4).

Definition 2.4.6. We will say that two maps $f: A \rightarrow B$ and $g: X \rightarrow Y$ are fiberwise orthogonal if they satisfy the equivalent properties of Proposition 2.4.5. We will denote this relation by $f \Perp g$ and say that $f$ is fiberwise left orthogonal to $g$, and that $g$ is fiberwise right orthogonal to $f$.

The intuitive idea behind this relation is that any fiber of $f$ is orthogonal to any fiber of $g$ in the external sense. This is the meaning of Condition (6) where "any fiber" has to be understood as "any pullback over an arbitrary base". Another way to understand fiberwise orthogonality is to say that it is the stabilization by base change of the relation $f \perp g$, which is the meaning of Condition (5).

Condition (7) helps to see that the relation $f \Perp g$ is stronger than the relation $f \perp g$ since, by Lemma 2.4.3. the latter only requires that $Z \times f$ is orthogonal to $g$ for every object $Z \in \mathcal{E}$. Thus,

$$
f \Perp g \quad \Rightarrow \quad f \Perp g \quad \Rightarrow \quad f \perp g .
$$

Remark 2.4.7. We have the following immediate observations:

1. If $f$ is fiberwise left orthogonal to $g$, then every base change $f^{\prime}$ of $f$ is left orthogonal to every base change $g^{\prime}$ of $g$. Moreover, $f \Perp g \Rightarrow f^{\prime} \Perp g^{\prime}$. 
2. The map $A \rightarrow 1$ for an object $A$ is fiberwise left orthogonal to a map $f: X \rightarrow Y$ if and only if it is internally left orthogonal to $f$. In particular two objects $A$ and $X$ are fiberwise orthogonal $(A \rightarrow 1) \Perp(X \rightarrow 1)$ if and only if they are internally orthogonal $(A \rightarrow 1) \perp(X \rightarrow 1)$.

\subsection{The fiberwise diagonal map}

We saw that the external and internal orthogonality of two maps $f$ and $g$ can be detected by the condition that some map $(\langle f, g\rangle$ or $\langle\langle f, g\rangle\rangle)$ be an isomorphism. The same thing is true for the fiberwise orthogonality, although the construction of the corresponding map is a bit more involved.

Definition 2.5.1. Take two maps $f: A \rightarrow B$ and $g: X \rightarrow Y$ in $\mathcal{E}$; pull them back to the common target $B \times Y$, i.e. consider the maps

$$
f_{B \times Y}=f \times \operatorname{id}_{Y}: A \times Y \rightarrow B \times Y
$$

and

$$
g_{B \times Y}=\operatorname{id}_{B} \times g: B \times X \rightarrow B \times Y
$$

and view them as objects over $B \times Y$. In the slice $\mathcal{E}_{/ B \times Y}$ one can form the $f_{B \times Y}$-diagonal of $g_{B \times Y}$ already used in 2.4.5. (3). We will denote this diagonal by $\{f, g\}$ and name it the fiberwise diagonal map.

$$
\{f, g\}=\Delta_{f_{B \times Y}}\left(g_{B \times Y}\right)=\left\langle\left\langle f_{B \times Y}, g_{B \times Y}\right\rangle_{B \times Y},\right.
$$

where the internal pullback hom on the right is taken in the topos $\mathcal{E}_{/ B \times Y}$. Explicitly,

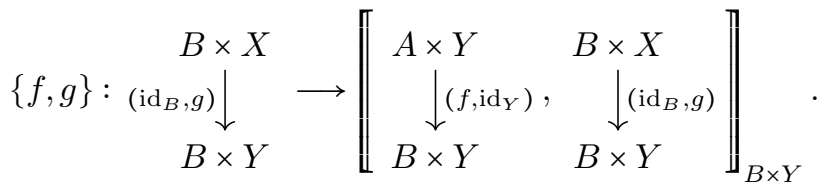

Remark 2.5.2. Let $b: 1 \rightarrow B$ and $y: 1 \rightarrow Y$ be points of $B$ and $Y$. We denote by $f_{b}$ and $g_{y}$ the corresponding fibers of $f$ and $g$. Since in a topos $\mathcal{E}$ colimits commute with base change, the fiber of $\{f, g\}$ at $(b, y)$ can be proven to be the diagonal map

$$
g_{y} \rightarrow \llbracket f_{b}, g_{y} \rrbracket .
$$

This is one of the reasons why we call this map the fiberwise diagonal map.

Proposition 2.5.3. Let $f$ and $g$ be maps in $\mathcal{E}$. Then $f \Perp g$ if and only if $\{f, g\}$ is an isomorphism.

Proof. This is exactly the content of 2.4.5(3).

We now arrive at our key technical result. 
Proposition 2.5.4. The following formula is true in any topos:

$$
\{f \square g, h\}=\{f,\{g, h\}\} .
$$

For the proof of this proposition we need the following two auxiliary lemmas.

Lemma 2.5.5. For all $A, C$ and $B \rightarrow C$ in any topos, the following square

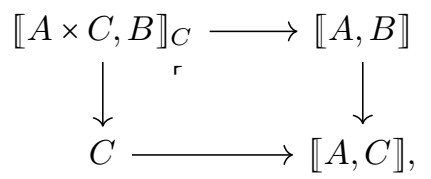

where $\llbracket-,-\rrbracket_{C}$ is the internal hom in $\mathcal{E}_{/ C}$ and where the bottom map is the diagonal map, is a pullback.

Proof. Using $C=\llbracket A \times C, C \rrbracket_{C}$ at the bottom left, we can factor the square as

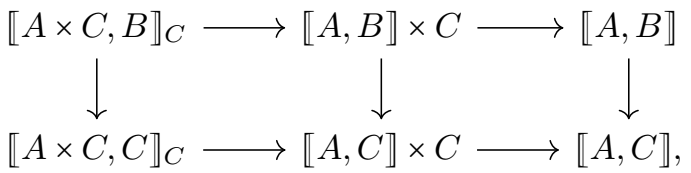

Then, the right square is obviously cartesian.

To prove that the left square is also cartesian we use first the fact that the base change $\mathcal{E} \rightarrow \mathcal{E}_{/ C}$ preserves internal homs; this shows that $\llbracket A \times C, B \times C \rrbracket_{C}=$ $\llbracket A, B \rrbracket \times C$. Then the left square is cartesian as the image of the cartesian square in $\mathcal{E}_{/ C}$

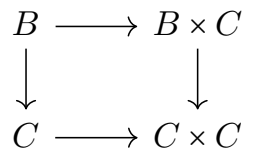

by the functor $\llbracket A \times C,-\rrbracket_{C}$ which preserves limits.

Lemma 2.5.6. The square

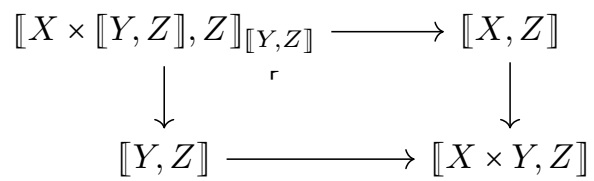

is a pullback. Hence, there is a canonical isomorphism

$$
\llbracket X \star Y, Z \rrbracket=\llbracket X \times \llbracket Y, Z \rrbracket, Z \rrbracket_{\llbracket Y, Z \rrbracket} .
$$

Proof. Setting $A=X, B=Z$ and $C=\llbracket Y, Z \rrbracket$ in the previous lemma we find that the square above is a pullback as claimed. Since the join is the pushout of the projections

$$
X \leftarrow X \times Y \rightarrow Y,
$$

the pullback of this square is canonically isomorphic to $\llbracket X \star Y, Z \rrbracket$. 
Proof of Proposition 2.5.4. We consider first the special case where the maps are of the following form

$$
f: X \rightarrow 1, g: Y \rightarrow 1, h: Z \rightarrow 1 .
$$

Then the map $\{f \square g, h\}$ becomes the $X \star Y$-diagonal of $Z$

$$
\{X \star Y \rightarrow 1, Z \rightarrow 1\}=Z \rightarrow \llbracket X \star Y, Z \rrbracket .
$$

On the other hand, the map $\{f,\{g, h\}\}$ becomes

$$
\{X \rightarrow 1, Z \rightarrow \llbracket Y, Z \rrbracket\}=Z \rightarrow \llbracket X \times \llbracket Y, Z \rrbracket, Z \rrbracket_{\llbracket Y, Z \rrbracket} .
$$

Lemma 2.5.6 shows that these two maps are the same. This proves our claim in the special case.

We prove the general case by arguing fiberwise, i.e. by viewing our maps as objects in the respective slice categories and then appealing to the special case above. We introduce the following convenient notation. First, we will denote the cartesian product of two objects $I$ and $J$ in $\mathcal{E}$ by concatenation $I J$. Then, for a map $f: X \rightarrow I$ in a topos $\mathcal{E}$, we will abuse notation and denote by $X$ the corresponding object in $\mathcal{E}_{/ I}$. If another object $J \in \mathcal{E}$ is given, we will denote by $X_{J}$ the base change of $X \in \mathcal{E}_{/ I}$ to $\mathcal{E}_{/ I J}$ along the projection $I \times J \rightarrow I$, i.e. $X_{J}$ is the map $X \times J \rightarrow I \times J$.

For two maps $f: X \rightarrow I$ and $g: Y \rightarrow J$, the map $f \square g$ in $\mathcal{E}$ corresponds to the object

$$
X_{J} \star Y_{I}
$$

in $\mathcal{E}_{/ I J}$, where the join is also computed in $\mathcal{E}_{/ I J}$. For a third object $K$, it is easy to compute that

$$
\left(X_{J} \star Y_{I}\right)_{K}=X_{J K} \star Y_{I K}
$$

in $\mathcal{E}_{/ I J K}$.

Similarly, for two maps $g: Y \rightarrow J$ and $h: Z \rightarrow K$, the map $\{g, h\}$ is defined as the map in $\mathcal{E}_{/ J K}$

$$
\left\langle\left\langle Y_{K} \rightarrow 1, Z_{J} \rightarrow 1\right\rangle\right.
$$

where the pullback hom is computed in $\mathcal{E}_{/ J K}$. For a third object $I \in \mathcal{E}$, because the pullback functor $\mathcal{E}_{/ J K} \rightarrow \mathcal{E}_{/ I J K}$ preserves exponentials, we have also

$$
\begin{aligned}
\left(\left\langle\left\langle Y_{K} \rightarrow 1, Z_{J} \rightarrow 1\right\rangle\right\rangle\right)_{I} & =\left(Z_{J} \rightarrow \llbracket Y_{K}, Z_{J} \rrbracket\right)_{I}=Z_{I J} \rightarrow \llbracket Y_{I K}, Z_{I J} \rrbracket \\
& =\left\langle\left\langle Y_{I K} \rightarrow 1, Z_{I J} \rightarrow 1\right\rangle\right\rangle
\end{aligned}
$$

in $\mathcal{E}_{/ I J K}$. Finally, we obtain the following canonical isomorphisms:

$$
\begin{array}{lll}
\{f \square g, h\} & \text { viewed as a map in } \mathcal{E}_{/ I J K} \\
=\left\langle\left\langle\left(X_{J} \star Y_{I}\right)_{K} \rightarrow 1, Z_{I J} \rightarrow 1\right\rangle\right\rangle & & \text { join in } \mathcal{E}_{/ I J}, \text { bracket in } \mathcal{E}_{/ I J K} \\
=\left\langle\left\langle X_{J K} \star Y_{I K} \rightarrow 1, Z_{I J} \rightarrow 1\right\rangle\right\rangle & & \text { computed in } \mathcal{E}_{/ I J K} \\
=\left\langle\left\langle X_{J K} \rightarrow 1,\left\langle\left\langle Y_{I K} \rightarrow 1, Z_{I J} \rightarrow 1\right\rangle\right\rangle\right\rangle\right. & & \text { special case applied to the topos } \mathcal{E}_{/ I J K} \\
=\left\langle\left\langle X_{J K} \rightarrow 1,\left(\left\langle\left\langle Y_{K} \rightarrow 1, Z_{J} \rightarrow 1\right\rangle\right\rangle\right)_{I}\right\rangle\right\rangle & & \text { inside bracket computed in } \mathcal{E}_{/ J K} \\
=\{f,\{g, h\}\} & & \text { viewed as a map in } \mathcal{E}_{/ I J K} .
\end{array}
$$




\subsection{Modalities and generalized Blakers-Massey theorems}

Given a class of maps $\mathcal{M}$ of $\mathcal{E}$, we write $\mathcal{M}^{\perp}$ for the class of maps which are externally right orthogonal to every map of $\mathcal{M}$. Similarly, the class ${ }^{\perp} \mathcal{M}$ denotes the class of maps externally left orthogonal to every map of $\mathcal{M}$.

Recall that a factorization system on a category $\mathcal{E}$ is the data of a pair $(\mathcal{L}, \mathcal{R})$ of classes of maps in $\mathcal{E}$ such that

1. every map $f$ in $\mathcal{E}$ can be factored in $f=r l$ where $l \in \mathcal{L}$ and $r \in \mathcal{R}$, and

2. $\mathcal{L}^{\perp}=\mathcal{R}$ and $\mathcal{L}={ }^{\perp} \mathcal{R}$.

In a factorization system, the right class is always stable by base change.

Definition 2.6.1. Let $\mathcal{E}$ be a topos. A modality on $\mathcal{E}$ is a factorization system $(\mathcal{L}, \mathcal{R})$ such that the left class $\mathcal{L}$ is also stable by base change.

Proposition 2.6.2. A factorization system $(\mathcal{L}, \mathcal{R})$ is a modality if and only if the stronger orthogonality property $\mathcal{L} \Perp \mathcal{R}$ holds.

Proof. The equivalence is given by Proposition 2.4.5.(7) which states exactly that the left class $\mathcal{L}$ is stable by base change.

An important source of modalities on a topos $\mathcal{E}$ are provided by the accessible left exact localizations of $\mathcal{E}$. (These are, in fact, exactly the subtopoi of $\mathcal{E}$, though we will not have occasion to use this observation.) To recall the construction, let $F^{\prime}: \mathcal{E} \rightarrow \mathcal{E}^{\prime}$ be a functor with fully-faithful right adjoint $i: \mathcal{E}^{\prime} \rightarrow \mathcal{E}$. As $i$ is fully-faithful, it is convenient to work with the associated endofunctor $F=i \circ F^{\prime}$, identifying $\mathcal{E}^{\prime}$ with its corresponding reflective subcategory in $\mathcal{E}$. We now have the following standard definitions:

Definition 2.6.3. Let $F: \mathcal{E} \rightarrow \mathcal{E}$ be as above.

1. A map $f: A \rightarrow B$ is said to be $F$-local if the square

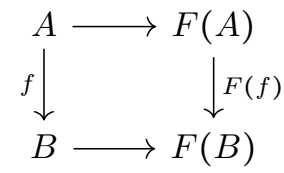

is cartesian.

2. A map $f: A \rightarrow B$ is an $F$-equivalence if $F(f)$ is an isomorphism.

Lemma 2.6.4. Let $F$ be a left exact localization of a topos $\mathcal{E}$. If we let $\mathcal{L}$ be the class of $F$-equivalences and $\mathcal{R}$ the class of $F$-local maps, then $(\mathcal{L}, \mathcal{R})$ forms a modality on $\mathcal{E}$. 
Proof. Given a map $f: A \rightarrow B$, one may produce directly a factorization $f=v \circ u$ by first applying $F$ and defining $C, u$ and $v$ by forming the pullback as in the following diagram

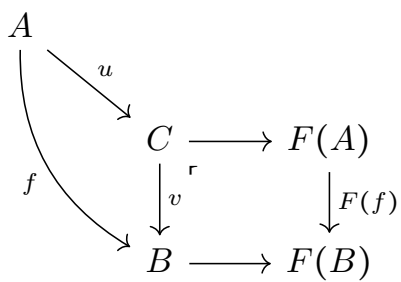

The map $v$ is $F$-local by construction, and one immediately checks that $u$ is an $F$-equivalence using the idempotence of $F$. That the class of $F$-equivalences is stable by base change is clear from the fact that $F$ preserves finite limits.

To check orthogonality we use the following observation. Let $g \in \mathcal{R}$ be any $F$-local map. Note that for any map $f$ we have

$$
\langle f, g\rangle=\langle f, F g\rangle=\langle F f, F g\rangle,
$$

where the first equality comes from the fact that $g$ is a base change of $F g$ and the second equality comes from the universal property of the localization $F$. It follows that if $f$ is an $F$-equivalence, so that $F(f)$ is an isomorphism, then $\langle F f, F g\rangle=\langle f, g\rangle$ is an isomorphism. This shows that $\mathcal{L} \subseteq{ }^{\perp} \mathcal{R}$ and $\mathcal{L}^{\perp} \supseteq \mathcal{R}$.

Now let $f: A \rightarrow B \in{ }^{\perp} \mathcal{R}$. We must show that $f$ is an $F$-equivalence. Consider the diagram

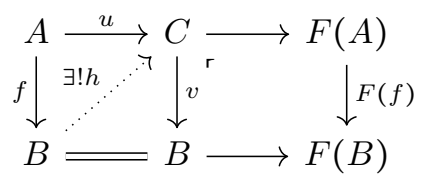

where $C, u$ and $v$ are defined by pullback. Since $v$ is $F$-local by construction, we have $f \perp v$. Hence we obtain a unique lift $h$. One can easily check that $F(h)$ provides an inverse to $F(f)$ which shows that $F(f)$ is an equivalence. This shows that $\mathcal{L}={ }^{\perp} \mathcal{R}$.

Finally, let $g: X \rightarrow Y \in \mathcal{L}^{\perp}$. Factor $g$ as $g=v \circ u$ as in the diagram

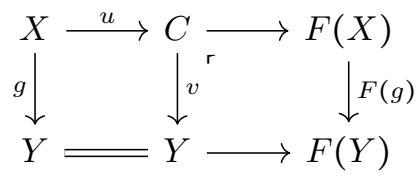

so that $v$ is $F$-local and $u$ is an $F$-equivalence by construction. We would like to show that the map $u$ is an isomorphism, as this implies that $g$ is $F$-local. But now, we have a unique lift $h$ in the following diagram

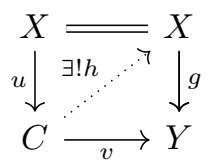


since $u \perp g$ by assumption and one readily checks that $h$ is the required inverse. This shows that $\mathcal{R}=\mathcal{L}^{\perp}$ and completes the proof.

Example 2.6.5. Goodwillie's $n$-excisive approximation construction $P_{n}$ is a left exact localization of the $\infty$-topos $[\mathcal{C}, \mathcal{S}]$ for some small category $\mathcal{C}$ with finite colimits and a terminal object. Hence, the $P_{n}$-equivalences and the $P_{n^{-}}$ local maps form a modality. This example is developed in detail Section 3, see Definition 3.1.5.

Let $(\mathcal{L}, \mathcal{R})$ on a topos $\mathcal{E}$ and suppose we are give a commutative square

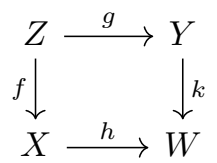

Definition 2.6.6. The square (1) is said to be $\mathcal{L}$-cartesian if the gap map

$$
(f, g): Z \rightarrow X \times{ }_{W} Y
$$

is in $\mathcal{L}$. The square is called $\mathcal{L}$-cocartesian if the cogap map

$$
\lfloor h, k\rfloor: X \cup_{Z} Y \rightarrow W
$$

is in $\mathcal{L}$.

Given a map $f: X \rightarrow Y$, its diagonal $\Delta f$ is the map

$$
\Delta f: X \rightarrow X \times_{Y} X
$$

induced by pulling back $f$ along itself. In particular $\Delta(X \rightarrow 1)$ is the classical diagonal $X \rightarrow X \times X$.

In [ABFJ17, the following two facts were proven about this situation:

Theorem 2.6.7 (Blakers-Massey [ABFJ17, Thm. 4.0.1]). Let Diagram (1) be a pushout square. Suppose that $\Delta f \square \Delta g \in \mathcal{L}$. Then the square is $\mathcal{L}$-cartesian.

Theorem 2.6.8 ("Dual" Blakers-Massey ABFJ17, Thm. 3.6.1]). Let Diagram (11) be a pullback square. Suppose that the map $h \square k \in \mathcal{L}$. Then the square is $\mathcal{L}$-cocartesian.

\section{The Goodwillie Localization}

We will now revisit the Goodwillie n-excisive localization from the perspective of topos theory. Our approach here is not the most general possible. In [BR14] a reasonably general framework for Goodwillie calculus in the language of model categories is developed. In [Heu15, the author constructs Goodwillie approximations of arbitrary categories. Here, however, we are particularly interested in functor categories, and more specifically, those valued in spaces. 


\subsection{The $n$-excisive modality}

All of our arguments can be carried out in the presheaf topos $[\mathcal{C}, \mathcal{S}]$ where $\mathcal{C}$ is a category with finite colimits and a terminal object, and hence we will work in that level of generality. We note that the standard examples of finite spaces (Fin) and finite pointed spaces $\left(\mathcal{F}_{i n}\right)$ fall into this category. Moreover, the class of such categories is closed under slicing and taking pointed objects. It includes in particular the source categories used by Goodwillie to construct the Goodwillie tower of a functor at a fixed object.

We stress that the target category is unpointed spaces because we rely on topos-theoretic arguments. No pointed category can be a non-trivial topos. However, our main results 3.3.4 3.4.2 and 3.4.1 are still valid for functors with values in pointed spaces. This follows from the observation that a natural transformation of functors to pointed spaces is $n$-excisive if and only it is still $n$-excisive after forgetting the basepoint, and analogously for $P_{n}$-equivalences.

Let us fix in this section a category $\mathcal{C}$ as above, writing 1 and 0 for the terminal and initial objects respectively. Recall that the starting point for Goodwillie calculus is the following

Definition 3.1.1. A functor $F: \mathcal{C} \rightarrow \mathcal{S}$ is $n$-excisive if it carries strongly cocartesian $(n+1)$-cubes in $\mathcal{C}$ to cartesian cubes in $\mathcal{S}$.

In order to provide examples of $n$-excisive functors, Goodwillie introduces the following construction. Given a functor $F: \mathcal{C} \rightarrow \mathcal{S}$, define a new functor $T_{n} F$ by the formula:

$$
T_{n} F(K):=\lim _{U \in P_{0}(\underline{n+1})} F(K \star U)
$$

There is a natural map

$$
t_{n} F: F \rightarrow T_{n} F
$$

determined at an object $K$ by the cartesian gap map of the cube $U \mapsto F(K \star U)$.

Remark 3.1.2. While we do not require that the category $\mathcal{C}$ admits finite products, the above formula nonetheless makes sense in our setting. Indeed, as $\mathcal{C}$ admits finite coproducts, it admits a tensoring over the category of finite sets by setting

$$
K \otimes U=\coprod_{U} K
$$

Since $\mathcal{C}$ has a terminal object, we can regard $U$ as an object of $\mathcal{C}$ by considering the object $1 \otimes U$. One can easily check that this makes $K \otimes U$ into a product in $\mathcal{C}$, so that one can define the join using the usual formula. Equivalently, one may define $K \star U$ directly by the colimit:

$$
K \star U=\operatorname{colim}\left\{\begin{array}{l}
\sum_{1} \searrow_{1}^{K} \searrow_{1} \\
\ldots
\end{array}\right.
$$

with $U$ copies of the terminal object appearing in the diagram. When $\mathcal{C}$ is taken to be $\mathcal{F} i n$ or $\mathcal{F} i n_{*}$, this definition coincides with the standard one. 
With this construction in hand, we now iterate, defining a functor $P_{n} F$ as the colimit of the induced sequence

$$
P_{n} F:=\operatorname{colim}\left\{F \rightarrow T_{n} F \rightarrow T_{n}^{2} F \rightarrow \cdots\right\}
$$

We summarize the relevant facts about this construction with the following

Proposition 3.1.3 (Goodwillie Goo03]). Let $F \in[\mathcal{C}, \mathcal{S}]$.

1. $P_{n} F$ is n-excisive.

2. The functor $P_{n}:[\mathcal{C}, \mathcal{S}] \rightarrow[\mathcal{C}, \mathcal{S}]$ commutes with finite limits.

3. The canonical map $F \rightarrow P_{n} F$ is universal for maps from $F$ to n-excisive functors. In particular, the functor $P_{n}$ is idempotent.

Proof. The proofs appearing in Goo03, as well as Rezk's streamlined version Rez13 are sufficiently general to go through in our setting with only minor modifications. Indeed, for a translation of these arguments into the language of $\infty$-categories, the reader may consult [Lur16] [Section 6.1.1].

Let us write $[\mathcal{C}, \mathcal{S}]^{(n)}$ for the full subcategory of $n$-excisive functors. The previous proposition can be summarized by asserting that the functor

$$
P_{n}:[\mathcal{C}, \mathcal{S}] \rightarrow[\mathcal{C}, \mathcal{S}]^{(n)}
$$

is a left exact localization Lur09, Prop. 5.2.7.4]. In particular, $[\mathcal{C}, \mathcal{S}]^{(n)}$ is itself a topos [Lur09, Prop. 6.1.0.1]).

Remark 3.1.4. The functor $P_{n}:[\mathcal{C}, \mathcal{S}] \rightarrow[\mathcal{C}, \mathcal{S}]$ commutes with filtered colimits. Since colimits in the localization $[\mathcal{C}, \mathcal{S}]^{(n)}$ are computed by reflecting the colimits of the ambient topos $[\mathcal{C}, \mathcal{S}]$ down to $[\mathcal{C}, \mathcal{S}]^{(n)}$ via $P_{n}$, the functor $P_{n}$ viewed as taking values in $[\mathcal{C}, \mathcal{S}]^{(n)}$ actually commutes with all colimits:

$$
P_{n} \operatorname{colim}_{i \in I} F_{i}=P_{n} \operatorname{colim}_{i \in I} P_{n} F_{i} .
$$

The right hand side is the colimit in $[\mathcal{C}, \mathcal{S}]^{(n)}$.

As is the case for any left exact localization, the functor $P_{n}$ determines two classes of maps via Definition 2.6.3. Moreover, according to Lemma 2.6.4, these two classes of maps form a modality.

Definition 3.1.5. We refer to the modality

$$
\text { ( } \left.P_{n} \text {-equivalences, } P_{n} \text {-local maps }\right)
$$

as the $n$-excisive modality.

Since the Generalized Blakers-Massey theorem of [ABFJ17 applies to an arbitrary modality on a topos, we may apply the result already at this point, using nothing but the left-exactness of the functor $P_{n}$. The statement obtained is the following: 
Proposition 3.1.6. Let

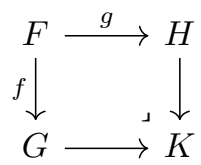

be a pushout square of functors. Suppose that $\Delta f \square \Delta g$ is a $P_{n}$-equivalence. Then so is the cartesian gap map

$$
(f, g): F \rightarrow G \times_{K} H
$$

We think the reader will agree that the statement in its current form is not entirely satisfactory: supposing that $f$ is a $P_{i}$-equivalence and $g$ is a $P_{j^{-}}$ equivalence, we would like a determination of $n$ in terms of $i$ and $j$. In the following sections, we will develop the tools to make such a calculation using the calculus of orthogonality developed above. The final result is the following.

Theorem 3.1.7. Let $f$ be a $P_{i}$-equivalence and $g$ a $P_{j}$-equivalence. Then the map

$$
\Delta f \square \Delta g
$$

is a $P_{i+j+1}$-equivalence.

\subsection{Cubical Diagrams and Orthogonality}

In order to prove Theorem 3.1.7, we are going to examine how the notion of cubical diagram in $\mathcal{C}$ is transformed by the Yoneda embedding

$$
y: \mathcal{C}^{\mathrm{op}} \rightarrow[\mathcal{C}, \mathcal{S}] .
$$

We will see that there is a close connection between strongly cocartesian cubical diagrams in $\mathcal{C}$ and fiberwise join products in $[\mathcal{C}, \mathcal{S}]$, leading to a number of useful characterizations of the classes of $P_{n}$-equivalences and $P_{n}$-local maps. From here, the calculus of orthogonality, and in particular the adjunction formula of Proposition 2.5.4 ultimately lead to the desired result. In the discussion which follows, we write

$$
R^{K}=\mathcal{C}(K,-)=y(K)
$$

for the representable functor determined by an object $K \in \mathcal{C}$. For a map $k: K \rightarrow$ $L$ we write

$$
R^{k}=y(k): \mathcal{C}(L,-) \rightarrow \mathcal{C}(K,-)
$$

for the induced map. We recall for later use that the Yoneda embedding preserves all limits and hence sends colimits in $\mathcal{C}$ to limits in $[\mathcal{C}, \mathcal{S}]$.

Now let $\mathcal{X}$ be a cubical diagram in $\mathcal{C}$ and let us put $K=\mathcal{X}(\varnothing)$. We denote by $\hat{x}$ the cubical diagram obtained by composition with the (contravariant) Yoneda embedding. That is, $\hat{x}=y \circ X$. The cocartesian gap map of this cube takes the form

$$
\Gamma(x): \underset{U \neq \varnothing}{\operatorname{colim}} R^{X(U)} \rightarrow R^{K}
$$

The interest in this map arises from the following elementary observation: 
Lemma 3.2.1. Let $F \in[\mathcal{C}, \mathcal{S}]$ be a functor. Then

$$
\Gamma(X) \perp(F \rightarrow 1) \Longleftrightarrow F \circ X \text { is cartesian }
$$

Proof. Unfolding the definition of the pullback hom $\langle\Gamma(X), F \rightarrow 1\rangle$ (and ignoring the trivial factors) we find

$$
\left\langle\ulcorner(X), F \rightarrow 1\rangle:\left[R^{X(\varnothing)}, F\right] \rightarrow\left[\operatorname{colim}_{U} R^{X(U)}, F\right]\right.
$$

But of course $\left[R^{X(\varnothing)}, F\right]=F(X(\varnothing))$ and

$$
\begin{aligned}
{\left[\operatorname{colim}_{U} R^{X(U)}, F\right] } & =\lim _{U}\left[R^{X(U)}, F\right] \\
& =\lim _{U} F(X(U))
\end{aligned}
$$

by Yoneda. Hence this is the map

$$
\left\langle\ulcorner(X), F \rightarrow 1\rangle: F(X(\varnothing)) \rightarrow \lim _{U} F(X(U))\right.
$$

which is an isomorphism exactly if the cube $F \circ X$ is cartesian.

Corollary 3.2.2. A functor $F \in[\mathcal{C}, \mathcal{S}]$ is n-excisive if and only if, for every strongly cocartesian $(n+1)$ cube $X$, we have $\Gamma(X) \perp F \rightarrow 1$.

In view of the previous corollary, it is natural to extend the definition of $n$-excisiveness to maps so that a functor is $n$-excisive if and only if the map $F \rightarrow 1$ is so. Concretely, we have

Definition 3.2.3. A map $f: F \rightarrow G$ of functors is said to be $n$-excisive if for all strongly cocartesian $(n+1)$-cubes $X$ we have $\Gamma(X) \perp f$.

For convenience we note that $f$ is $n$-excisive if and only if for all strongly cocartesian $X$ as above, the square

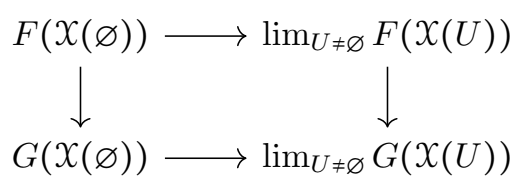

is a pullback.

The following construction is a useful source of strongly cocartesian diagrams. The reader may wish to compare [BJM15, Example 2.8] where a similar construction is considered.

Construction 3.2.4. Let $\left\{k_{i}: K_{i} \rightarrow L_{i}\right\}_{i=1}^{n}$ be a family of maps in $\mathcal{C}$. For $U \subseteq \underline{n}$, define

$$
\sigma_{U}\left(k_{i}\right)= \begin{cases}K_{i} & i \notin U \\ L_{i} & i \in U\end{cases}
$$


Associated to the family $\left\{k_{i}\right\}$ is a $n$-cubical diagram $\mathcal{K}$ defined by the formula

$$
\mathcal{K}(U)=\bigsqcup_{1 \leq i \leq n} \sigma_{U}\left(k_{i}\right)
$$

where for $U \subseteq V$, the induced map $\mathcal{K}(U) \rightarrow \mathcal{K}(V)$ is given by

$$
\mathcal{K}(U \hookrightarrow V)= \begin{cases}k_{i} & i \in V \backslash U \\ \operatorname{id}_{\sigma_{V}(i)} & \text { otherwise }\end{cases}
$$

Lemma 3.2.5. For any family of maps $\left\{k_{i}: K_{i} \rightarrow L_{i}\right\}_{i=1}^{n}$ the cubical diagram $\mathcal{K}$ is strongly cocartesian.

Proof. In the notation of Definition 2.2.1, we have $\mathcal{K}=k_{1} \boxplus \cdots \boxplus k_{n}$.

Lemma 3.2.6.

$$
\Gamma(\mathcal{K})=R^{k_{1}} \square \cdots \square R^{k_{n}}
$$

Proof. We have $\hat{\mathcal{K}}:=y \circ \hat{\mathcal{K}}=R^{k_{1}} \otimes \cdots \otimes R^{k_{n}}$, since the Yoneda functor takes coproduct to product. Hence the cocartesian gap map of $\hat{\mathcal{K}}$ is equal to $R^{k_{1}} \square$ $\cdots \square R^{k_{n}}$.

Example 3.2.7. Suppose the category $\mathcal{C}$ is pointed, that is, that the initial and terminal objects coincide in $\mathcal{C}$. It will be convenient in this case to write $\vee$ for the coproduct in $\mathcal{C}$ in order to make contact with the traditional notation. In particular, we have $K \vee 1=K$ for all objects $K \in \mathcal{C}$.

Now consider a family of objects $\left\{K_{i}\right\}_{i=1}^{n}$ in C. Applying Construction 3.2 .4 to the collection of maps $\left\{K_{i} \rightarrow 1\right\}_{i=1}^{n}$ we find that the resulting cube may be more simply described as

$$
\mathcal{K}(U)=\bigvee_{i \notin U} K_{i}
$$

Now let $F: \mathcal{C} \rightarrow \mathcal{S}$ be a functor. Unraveling the definition shows that the pullback hom $\langle\ulcorner(\mathcal{K}), F\rangle$ is the map

$$
F\left(\bigvee_{i} K_{i}\right) \rightarrow \lim _{U \neq \varnothing} F\left(\bigvee_{i \notin U} K_{i}\right)
$$

The fiber of this map is what Goodwillie refers to as the $n$-th cross-effect, writing $\left(c r_{n} F\right)\left(K_{1}, \ldots, K_{n}\right)$. It follows immediately from these considerations that we have $\Gamma(\mathcal{K}) \perp(F \rightarrow 1)$ for every family $\left\{K_{i}\right\}_{i=1}^{n}$ of objects of $\mathcal{C}$ if and only if $F$ is of degree $(n-1)$ in the sense of [BJM15, Definition 3.21]; that is, $c r_{n} F$ vanishes.

It is well known that to be of degree $n$ is strictly weaker than to be $n$-excisive. Nonetheless, we will show below that we can recover the notion of $n$-excisiveness from the cubical diagrams $\mathcal{K}$ by replacing the external orthogonality relation $\perp$ by the stronger fiberwise orthogonality relation $\Perp$. It is exactly this observation which motivates the introduction of this stronger notion. 
Construction 3.2.4 turns out to be quite general: in fact, as we now show, every cubical diagram can be obtained from it after a single cobase change. To make this precise, suppose we are given a strongly cocartesian cube $\mathcal{X}: P(\underline{n}) \rightarrow$ C. Let us put $K=P(\varnothing)$ and $K_{i}=P(\{i\})$. The functorial action of $\mathcal{X}$ gives us maps

$$
k_{i}:=X(\varnothing \rightarrow\{i\}): K \rightarrow K_{i} .
$$

Applying Construction 3.2.4 we obtain a new cubical diagram which, in this case, we will denote by $X_{\square}$ (the notation being inspired by Lemma 3.2.6 above). Unwinding the definition, we find that

$$
X_{\square}(\varnothing)=K^{\sqcup n}
$$

so that the codiagonal $\nabla: K^{\sqcup n} \rightarrow K$ provides a canonical map $X_{\square}(\varnothing) \rightarrow X(\varnothing)$.

Lemma 3.2.8. The strongly cocartesian cube $\mathcal{X}$ is obtained from $X_{\square}$ by cobase change along the codiagonal map

$$
\nabla: K^{\sqcup n} \rightarrow K
$$

Proof. The lemma asserts that for any $U \subseteq \underline{n}$, the square

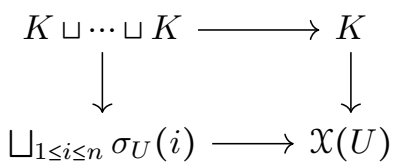

is a pushout. But since $X$ is strongly cocartesian, we have that

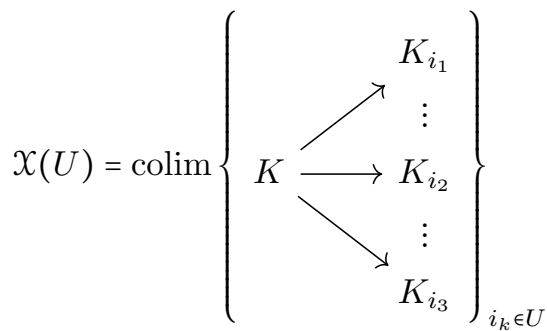

and one easily sees that this coincides with the pushout above by a simple cofinality argument.

It is immediate from the previous lemma and the fact that the Yoneda embedding sends colimits in $\mathcal{C}$ to limits in $[\mathcal{C}, \mathcal{S}]$ that the corresponding cube of representable functors $\hat{X}$ is obtained from $\hat{X}_{\square}$ by base change along the $n$-fold diagonal map

$$
\Delta: R^{X(\varnothing)} \rightarrow\left(R^{X(\varnothing)}\right)^{\times n}=R^{X_{\square}(\varnothing)}
$$

It will be convenient in what follows to introduce special notation for the cocartesian gap maps of these two cubes. We will use this notation exclusively in 
the case where the given cubical diagram $X$ is known to be strongly cocartesian. In this case, the cocartesian gap map of the cube $\hat{X}$ will be denoted

$$
\gamma^{x}: \Gamma^{x} \rightarrow R^{x(\varnothing)}
$$

where $\Gamma^{x}:=\operatorname{colim}_{U \neq \varnothing} R^{x(U)}$. For $\hat{X}_{\square}$, on the other hand, we will write

$$
w^{x}: W^{x} \rightarrow\left(R^{x(\varnothing)}\right)^{\times n}
$$

with $W^{x}$ defined by the analogous colimit for the cube $X_{\square}$. Some justification for this special notation will be given in Remark 3.2 .11 below. For now we observe

Lemma 3.2.9. For any strongly cocartesian cube $\mathcal{X}$ in $\mathcal{C}$, the square

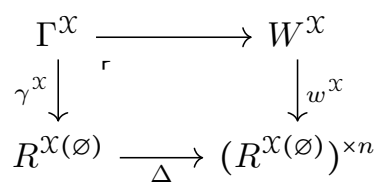

is a pullback in $[\mathcal{C}, \mathcal{S}]$.

Proof. Immediate since colimits in $[\mathcal{C}, \mathcal{S}]$ are stable by base change.

Combining Lemma 3.2 .6 with the definition of the fiberwise join, we deduce immediately that

Lemma 3.2.10. For any strongly cocartesian cubical diagram $\mathcal{X}$ in $\mathcal{E}$, the cocartesian gap map $\gamma^{x}$ of the cube of representable functors $\hat{x}$ is given by the expression

$$
\gamma^{X}=R^{k_{1}} \star_{R^{K}} \cdots \star_{R^{K}} R^{k_{n}}
$$

where $k_{i}=X(\varnothing \rightarrow\{i\}): K \rightarrow K_{i}$.

The above discussion has an important special case, which we now describe. Note that a given strongly cocartesian diagram $X$ is completely determined by the family of maps

$$
\left\{f_{i}: X(\varnothing) \rightarrow X(\{i\})\right\}_{1 \leq i \leq n}
$$

Consequently, we may identify the category of strongly cocartesian $n$-cubes $X$ such that $X(\varnothing)=K$ with the $n$-th cartesian power of the coslice category $\left(\mathcal{C}_{K /}\right)^{\times n}$. As $\mathcal{C}$ has a terminal object, this category clearly has one as well, an $n$-cube which we will denote by $\mathcal{T}_{n}^{K}$ and which is determined by $\mathcal{T}_{n}^{K}(\varnothing)=K$ and $\mathcal{T}_{n}^{K}(\{i\})=1$ for $1 \leq i \leq n$. More generally, the reader can easily check that we have

$$
\mathcal{T}_{n}^{K}(U)=K \star U
$$

in the sense of Remark 3.1.2 Applying Yoneda as in the proof of 3.2 .1 we find that

$$
\left\langle\gamma^{\mathcal{T}_{n}^{K}}, F\right\rangle=\left(t_{n} F\right)(K): F(K) \rightarrow \lim _{U} F(K \star U)
$$


is Goodwillie's map $t_{n} F$ introduced in the previous section. As these distinguished strongly cocartesian cubes play a central role in the theory and are entirely determined by the given object $K$, it will be convenient to use the abbreviation

$$
\gamma_{n}^{K}: \Gamma_{n}^{K} \rightarrow R^{K}
$$

and

$$
w_{n}^{K}: W_{n}^{K} \rightarrow\left(R^{K}\right)^{\times n}
$$

for the maps $\gamma^{\mathcal{T}_{n}^{K}}$ and $w^{\mathcal{T}_{n}^{K}}$ constructed above. Note that by construction

$$
w_{n}^{K}=\left(w_{1}^{K}\right)^{\square n}
$$

where $w_{1}^{K}=R^{K \rightarrow 1}: R^{1} \rightarrow R^{K}$. In this case, then the statement of Lemma 3.2.9 asserts that the square

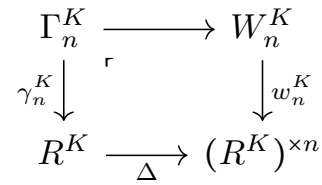

is a pullback for any $K \in \mathcal{C}$.

Remark 3.2.11. The pullback diagram (2) is analogous to a well-known construction in classical homotopy theory. For a pointed space $(X, x)$, the $n$-fold fat wedge of $X$, denoted $W_{n}(X)$ may be defined as the iterated pushout product

$$
\underset{X^{\times n}}{W_{n}(X)}:=\left(\begin{array}{c}
1 \\
x \downarrow \\
w_{n} \\
X
\end{array}\right)^{\square n} .
$$

Note that it comes equipped with a canonical inclusion $w_{n}$ into the $n$-fold product as shown. The pullback of this map along the diagonal $X \rightarrow X^{\times n}$ is known as the $n$-th Ganea fibration, and denoted $\Gamma_{n}(X)$.

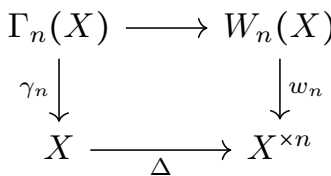

Recall from Subsection 2.3 that the pullback of an $n$-fold pushout product along the diagonal map is called the $n$-fold fiberwise join. Thus the map $\gamma_{n}$ may alternatively be described as

$$
\gamma_{n}=1 \star_{X} \cdots \star_{X} 1
$$

From the discussion of the fiberwise join, then, it is immediately clear that the fiber of the map $\gamma_{n}$ has the description

$$
\operatorname{fib}_{x} \gamma_{n}=(\Omega X)^{\star n}
$$


as is well known.

In fact, this construction makes sense in any topos. Returning to the situation at hand, when the category $\mathcal{C}$ is pointed, we find that the representable functor $R^{1}$ is in fact the terminal functor in $[\mathcal{C}, \mathcal{S}]$. Hence for any object $K \in \mathcal{C}$, the terminal map $K \rightarrow 1$ provides the representable functor $R^{K}$ with a canonical base point

$$
R^{1} \rightarrow R^{K}
$$

Examining the pullback diagram (21) above, we find that it is exactly the $n$-th Ganea fibration of the representable $R^{K}$ as calculated in the topos [ $\mathcal{C}, \mathcal{S}$ ], which is the justification for the notation introduced above. From this perspective, Theorem 3.3.1 (4) below may be read as saying that the Goodwillie localization of the functor category $[\mathcal{C}, \mathcal{S}]$ is obtained by inverting the $n$-th Ganea fibration of the representable $R^{K}$ for all $K \in \mathcal{C}$.

Let us also point out that diagram (2) is well-defined and still a pullback even if $\mathcal{C}$ is not pointed.

\subsection{A Characterization of $n$-excisive maps}

We now proceed to give a number of characterizations of the class of $n$-excisive maps as defined above. The reader will perhaps not be surprised to learn that they coincide with the $P_{n}$-local maps determined by the localization functor $P_{n}:[\mathcal{C}, \mathcal{S}] \rightarrow[\mathcal{C}, \mathcal{S}]$, though this is not a priori obvious. Furthermore, characterization (2) in the following theorem provides the main tool for establishing the compatibility of $P_{n}$-equivalences with the pushout product.

Theorem 3.3.1. Let $f: F \rightarrow G$ be a map in $[\mathcal{C}, \mathcal{S}]$. The following statements are equivalent:

(1) For every family of maps $\left\{h_{i}: K_{i} \rightarrow L_{i}\right\}_{i=0}^{n}$ in $\mathcal{C}$ we have

$$
R^{h_{0}} \square \cdots \square R^{h_{n}} \Perp f
$$

(2) For all $K \in \mathcal{C}$ we have $w_{n+1}^{K} \Perp f$.

(3) For every family of maps $\left\{h_{i}: K \rightarrow K_{i}\right\}_{i=0}^{n}$ in $\mathcal{C}$ we have

$$
R^{K_{0}} \star_{R^{K}} \cdots \star_{R^{K}} R^{K_{n}} \perp f
$$

(4) For all $K \in \mathcal{C}$ we have $\gamma_{n+1}^{K} \perp f$.

(5) The map $f$ is $P_{n}$-local.

(6) The map $f$ is n-excisive.

Proof. We will begin with the equivalences $(3) \Leftrightarrow(4) \Leftrightarrow(5) \Leftrightarrow(6)$.

$(3) \Rightarrow(4)$ This is the special case $K_{i}=1$ for all $i$ 
$(4) \Rightarrow(5)$ Examining the definition, we find that $\left\langle\gamma_{n+1}^{K}, F\right\rangle$ is the cartesian gap map of the commutative square

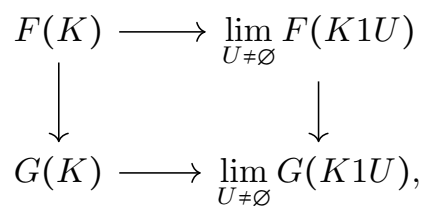

Hence if $\gamma_{n+1}^{K} \perp f$, this square is a pullback. Recognizing the right vertical map as $T_{n}(f)$, it follows that $f$ is a pullback of $T_{n}(f)$. But then it is a pullback of all composites $T_{n}^{k} f$ because $T_{n}$ preserves finite limits. Since finite limits commute with filtered colimits in $\mathcal{S}, f$ is a pullback of $P_{n} f=\operatorname{colim}_{k} T_{n}^{k} f$, ie. $f$ is $P_{n}$-local.

$(5) \Rightarrow(6)$ Now assume that $f$ is $P_{n}$-local and let $X$ be a strongly cocartesian $(n+1)$-cube. Write $K=X(\varnothing)$. Consider the following commutative diagram:

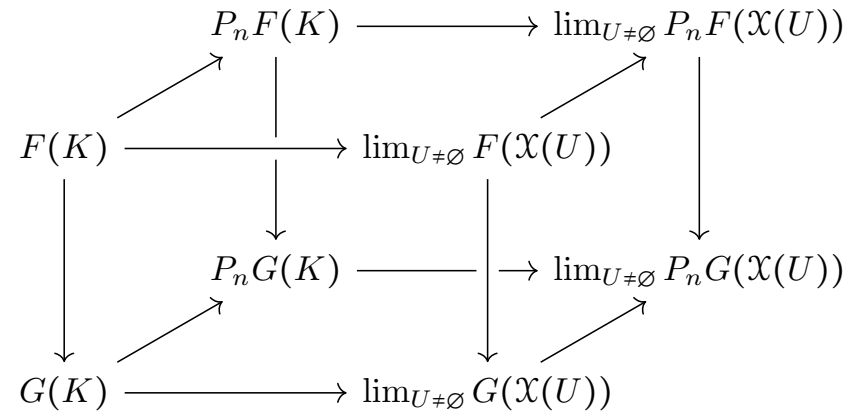

We need to show that the front face is a pullback (see Definition 3.2.3) . The right and left faces are a pullbacks by assumption. The back square is trivially a pullback: both horizontal maps are isomorphisms because $P_{n} F$ and $P_{n} G$ are $n$-excisive functors by Proposition 3.1.3 Thus, the composite diagonal square is a pullback. Hence, the front is also a pullback.

$(6) \Rightarrow(3)$ According to Lemma 3.2.10, the cocartesian gap map of any strongly cocartesian diagram can be expressed in this form. Hence if $f$ is $n$ excisive, it is orthogonal to such a map by definition.

We now treat statements (1) and (2).

$(1) \Rightarrow(2)$ This is the special case where $h_{i}=K \rightarrow 1$ for all $i$.

$(2) \Rightarrow(3)$ We have seen above that there is a pullback square

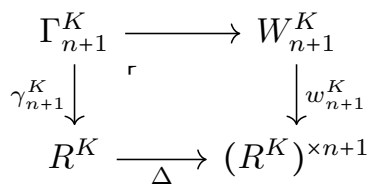

for any $K \in \mathcal{C}$. But by the Definition 2.4.6 of fiberwise orthogonality, or more precisely by Proposition 2.4.5(7), $f$ is orthogonal to any pullback of the map $w_{n+1}^{K}$, in particular $\gamma_{n+1}^{K}$ as claimed. 
(6) $\Rightarrow(1)$ Lemma 3.2.6 identifies the map

$$
R^{h_{0}} \square \cdots \square R^{h_{n}}
$$

as the cocartesian gap map of the strongly cocartesian cube determined by Construction 3.2.4 and so the relation $R^{h_{0}} \square \cdots \square R^{h_{n}} \perp f$ holds by definition. It remains to show that $f$ is orthogonal to any base change of this map. But since we already know $(6) \Rightarrow(5), f$ is $P_{n}$-local. The result now follows since $P_{n}$-equivalences are stable by base change.

Remark 3.3.2. It is not possible to replace the fiberwise orthogonality relation $\Perp$ it items (1) and (2) with the weaker external orthogonality relation $\perp$. Indeed, as pointed out in Example 3.2.7 the latter notion detects functors which are of degree $n$, a strictly weaker condition.

Remark 3.3.3. In ABFJ17 we deduce the classical Blakers-Massey theorem from our generalized version by using the fact that the $n$-connected $/ n$-truncated modalities are generated by pushout product powers of the map $S^{0} \rightarrow 1$. Theorem 3.3 .1 (2) states that in the same sense the Goodwillie tower, that is the $n$-excisive modalities, are generated by the pushout product powers of the maps $w_{1}^{K}: R^{1} \rightarrow R^{K}$ for all $K$ in $\mathrm{C}$.

We can now prove the main result of this section. Recall the fiberwise diagonal $\{f, g\}$ of two maps $f$ and $g$ as defined in 2.5.1. By Proposition 2.5.3 it is an isomorphism if and only if the maps $f$ and $g$ are fiberwise orthogonal. A crucial role in the proof of the next theorem is played by the formula $\{f \square g, h\}=\{f,\{g, h\}\}$ demonstrated in Proposition 2.5.4. It allows us to use adjunction tricks for fiberwise orthogonality. The reader is invited to compare the next theorem with ABFJ17, Cor. 3.15] where the $n$-connected $/ n$-truncated modalities for $n \geq-2$ are treated.

Theorem 3.3.4. Let $f$ be a $P_{m}$-equivalence, $g$ a $P_{n}$-equivalence and $h$ a $p$ excisive map. Then:

(1) The map $\left\{w_{n}^{K}, h\right\}$ is $(p-n)$-excisive

(2) The map $f \square w_{n}^{K}$ is a $P_{n+m}$-equivalence

(3) The map $\{f, h\}$ is $(p-m-1)$-excisive

(4) The map $f \square g$ is a $P_{m+n+1}$-equivalence

Proof. (1). It is immediate from Lemma 3.2.6 that

$$
w_{p+1}^{K}=w_{p-n+1}^{K} \square w_{n}^{K}
$$

Therefore, by Proposition 2.5.4 we have

$$
\left\{w_{p+1}^{K}, h\right\}=\left\{w_{p-n+1}^{K},\left\{w_{n}^{K}, h\right\}\right\}
$$


for all $K \in \mathcal{C}$. The map on the left is an isomorphism by the assumption that $h$ is $p$-excisive, and hence so is the one on the right. Theorem 3.3.1 (2) then gives the desired result.

(2). If $k$ is any $(n+m)$-excisive map, we have

$$
\left\{f \square w_{n}^{K}, k\right\}=\left\{f,\left\{w_{n}^{K}, k\right\}\right\}
$$

But the map $\left\{w_{n}^{K}, k\right\}$ is $m$-excisive by (1).

(3). Again by Theorem 3.3.1 (2), it suffices to check that the map $\left\{w_{p-m}^{K},\{f, h\}\right\}$ is an isomorphism for any $K \in \mathcal{C}$. But

$$
\left\{w_{p-m}^{K},\{f, h\}\right\}=\left\{w_{p-m}^{K} \square f, h\right\}
$$

and since $w_{p-m}^{K} \square f$ is $p$-excisive by (2), the right map is an isomorphism.

(4). Let $k$ be any $(m+n+1)$-excisive map. Then since

$$
\{f \square g, k\}=\{f,\{g, k\}\}
$$

and the map $\{g, k\}$ is $(m+n+1)-n-1=m$ excisive, the result follows.

The compatibility of the Goodwillie tower with the pushout product stated in Theorem 3.3.4 (4) is what we are really after. It will allow us to prove the Blakers-Massey analogue for the Goodwillie tower. One direct application is

Example 3.3.5. Recall that a functor $F$ is $m$-reduced if the map $F \rightarrow 1$ is a $P_{m-1}$-equivalence. Let $F$ be $m$-reduced and $G$ be $n$-reduced. Then the map

$$
(1 \rightarrow F) \square(1 \rightarrow G)=(F \vee G \rightarrow F \times G)
$$

is a $P_{m+n-1}$-equivalence. Taking the cofiber it follows that $F \wedge G$ is $(m+n-1)$ reduced because, as a left class, $P_{n}$-equivalences are stable by cobase change. Similarly, the map

$$
(F \rightarrow 1) \square(G \rightarrow 1)=(F \star G \rightarrow 1)
$$

is a $P_{m+n-1}$-equivalence, i.e. $F \star G$ is $(m+n-1)$-reduced.

\subsection{The Blakers-Massey Theorem for the Goodwillie Tower}

Theorem 3.4.1 (Blakers-Massey theorem for Goodwillie Calculus). Let

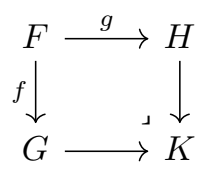

be a pushout square of functors. If $f$ is a $P_{m}$-equivalence and $g$ is a $P_{n^{-}}$ equivalence, then the induced map

$$
(f, g): F \rightarrow G \times_{K} H
$$

is a $P_{m+n+1}$-equivalence. 
Proof. If a map $h$ is $k$-excisive then its diagonal $\Delta h$ is also $k$-excisive because $P_{k}$ is left exact. Theorem 3.3.4(4) then implies that $\Delta f \square \Delta g$ is a $P_{m+n+1^{-}}$ equivalence: $\Delta f \square \Delta g$ is in the left class of the modality associated to $P_{m+n+1}$. Now we apply the Theorem 2.6.7 and learn that $(f, g)$ is in the same left class. Hence, the gap map is a $P_{m+n+1}$-equivalence.

Theorem 3.4.2 ("Dual" Blakers-Massey theorem for Goodwillie Calculus). Let

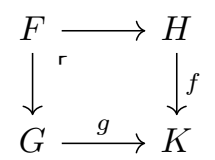

be a pullback square of functors. If $f$ is a $P_{m}$-equivalence and $g$ is a $P_{n^{-}}$ equivalence, then the cogap map

$$
\lfloor f, g\rfloor: G \sqcup_{F} H \rightarrow K
$$

is a $P_{m+n+1}$-equivalence.

Proof. By Theorem 3.3.4(4) the map $f \square g$ is a $P_{m+n+1}$-equivalence. By Theorem 2.6.8 the same holds for the cogap $\lfloor f, g\rfloor$.

\subsection{Delooping theorems}

In this section, we rederive some of the fundamental delooping results of Goo03. To begin, let us recall the following definition:

Definition 3.5.1. Let $f: X \rightarrow Y$ be a map in a topos $\mathcal{E}$. We say that $f$ is a principal fibration if there exists an object $B \in \mathcal{E}$, map $h: Y \rightarrow B$ and cover $b: 1 \rightarrow B$ such that the square

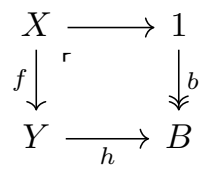

is cartesian.

Fix a functor $F: \mathcal{C} \rightarrow \mathcal{S}$. It is easily checked that if $F$ is $k$-excisive, then it is $n$-excisive for any $n \geq k$. Hence the universal property of $P_{n}$ implies that for any $k \leq n$ we have a canonical map $q_{n, k}: P_{n} F \rightarrow P_{k} F$. They form the Goodwillie tower of $F$. The map $q_{n, k}$ is a $P_{k}$-equivalence by construction. We will use the abbreviation

$$
q_{n}=q_{n, n-1}: P_{n} F \rightarrow P_{n-1} F .
$$

Now let us denote by $C$ the pushout of the following diagram

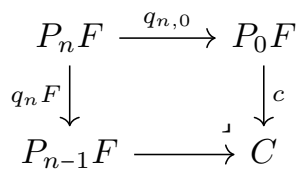


in $[\mathcal{C}, \mathcal{S}]$. We obtain an induced map $\left\lfloor q_{n-1,0}, \operatorname{id}_{P_{0} F}\right\rfloor: C \rightarrow P_{0} F$ so that we may regard the above diagram as living in the slice category $[\mathcal{C}, \mathcal{S}]_{/ P_{0} F}$. Note that $c$ is a $P_{n-1}$-equivalence because, as a left class of a factorization system, $P_{n-1^{-}}$ equivalences are closed by cobase change. Clearly $\operatorname{id}_{P_{0} F}$ is a $P_{n-1}$-equivalence. So $C \rightarrow P_{0} F$ is also a $P_{n-1}$-equivalence. More vaguely stated, $C$ is pointed and $n$-reduced relative to the constant functor $P_{0} F$.

Applying $P_{n}$ to the square (3) one obtains the induced square

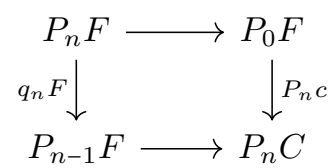

in $[\mathcal{C}, \mathcal{S}]_{/ P_{0} F}^{(n)}$. By Remark 3.1 .4 this is still a pushout in $[\mathcal{C}, \mathcal{S}]_{/ P_{0} F}^{(n)}$, but more is true.

Theorem 3.5.2. The square (4) is cartesian and the map

$$
q_{n}: P_{n} F \rightarrow P_{n-1} F
$$

is a principal fibration in the topos $[\mathcal{C}, \mathcal{S}]_{P_{0} F}^{(n)}$.

Proof. To see that the square is cartesian, it suffices to work in the ambient topos $[\mathcal{C}, \mathcal{S}]$, since the forgetul functors $[\mathcal{C}, \mathcal{S}]_{/ P_{0} F}^{(n)} \rightarrow[\mathcal{C}, \mathcal{S}]^{(n)} \rightarrow[\mathcal{C}, \mathcal{S}]$ preserves and reflects pullbacks. The map $q_{n, 0}$ is a $P_{0}$-equivalence and $q_{n}$ is a $P_{n-1^{-}}$ equivalence. Hence, applying Theorem 3.4.1 we find that the cartesian gap map $P_{n} F \rightarrow P_{n-1} F \times_{P_{n} C} P_{0} F$ is a $P_{n}$-equivalence. But since the source and target of this map are $n$-excisive, the gap map is an isomorphism. Thus the square 4 is cartesian as claimed.

Unwinding the definition of principal fibration given above in the slice category $[\mathcal{C}, \mathcal{S}]_{/ P_{0} F}^{(n)}$, we find that it remains to verify that the map $P_{n} c$ is a cover. In fact, it suffices to check the statement in the category $[\mathcal{C}, \mathcal{S}]^{(n)}$. By Proposition A.0.5 it suffices to check that the map $P_{n} c$ is a $P_{0}$-equivalence. But it is even a $P_{n-1}$-equivalence since $c$ is, as we have already observed.

If the category $\mathcal{C}$ is pointed, there exists a canonical map $F(1) \rightarrow F(X)$ for any $X \in \mathcal{C}$. This induces maps $P_{0} F \rightarrow F \rightarrow P_{n} F$. We define $D_{n} F$, the $n$-th homogeneous layer of $F$, by the pullback square

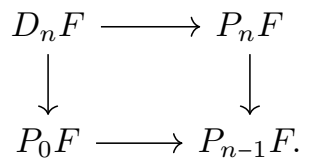

Corollary 3.5.3. The functor $P_{n} C$ is a delooping of $D_{n} F$ in the categories $[\mathcal{C}, \mathcal{S}]_{/ P_{0} F}$ and $[\mathcal{C}, \mathcal{S}]_{/ P_{0} F}^{(n)}$ in the sense that $D_{n} F=\Omega_{P_{0} F} P_{n} C$. 
This corollary yields a proof of Goodwillie's delooping result for homogeneous functors independent of Section 2 of his paper [Goo03].

Proof. We have the following commutative diagram

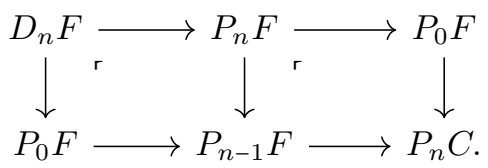

where both squares are pullbacks. We deduce that $D_{n} F=\Omega_{P_{0} F} P_{n} C$ where $\Omega_{P_{0} F}$ denotes the loop functor in the category $[\mathcal{C}, \mathcal{S}]_{/ P_{0} F}$. Moreover, as every object in the above diagram is $n$-excisive, we may regard the diagram as living in the subcategory $[\mathcal{C}, \mathcal{S}]_{/ P_{0} F}^{(n)}$, and since the inclusion $[\mathcal{C}, \mathcal{S}]_{/ P_{0} F}^{(n)} \hookrightarrow[\mathcal{C}, \mathcal{S}]_{/ P_{0} F}$ is fully-faithful and preserves limits, the second assertion follows as well.

Theorem 3.5.4. Consider a cocartesian square

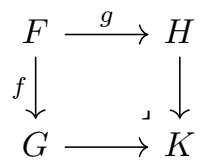

in $[\mathcal{C}, \mathcal{S}]$ where $f$ and $g$ are $P_{n}$-equivalences and $F, G$ and $H$ are $(2 n+1)$-excisive. Then the induced square

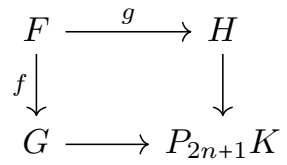

is cartesian in $[\mathcal{C}, \mathcal{S}]$.

Proof. By Theorem 3.4.1 the gap

$$
(f, g): F \rightarrow G \times_{K} H
$$

is a $P_{2 n+1}$-equivalence. The comparison map

$$
G \times{ }_{K} H \rightarrow G \times \times_{P_{2 n+1} K} H
$$

induced by $K \rightarrow P_{2 n+1} K$ is also a $P_{2 n+1}$-equivalence. So their composition is a $P_{2 n+1}$-equivalence between $(2 n+1)$-excisive functors. Hence it is an isomorphism.

Corollary 3.5.5. (Arone-Dwyer-Lesh [ADL08, Thm. 4.2]) For every n-reduced functor $F$ the canonical map

$$
P_{2 n-1} F \rightarrow \Omega P_{2 n-1} \Sigma F
$$

is an isomorphism. If $F$ is also $(2 n-1)$-excisive it is infinitely deloopable. 
The assertion follows from Theorem 3.5.4 but we going to give a direct proof.

Proof. The isomorphism follows by applying Theorem 3.4.1 to the pushout square

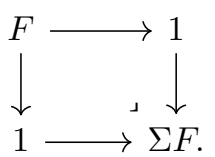

Since the class of $P_{n-1}$-equivalence is stable by colimits in $\mathcal{E}^{\rightarrow}$, the functor $\Sigma F$ is $n$-reduced when $F$ is. The theorem may then be iterated by taking $P_{2 n-1} \Sigma F$ in place of $F$.

Theorem 3.5.6. Consider a cartesian square

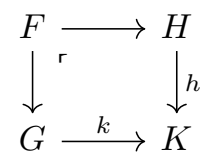

in $[\mathcal{C}, \mathcal{S}]$ where $h$ and $k$ are $P_{n}$-equivalences and $G, H$ and $K$ are $(2 n+1)$ excisive. Then the cogap map $\lfloor h, k\rfloor$ is a $P_{2 n+1}$-equivalence and the square is cocartesian in $[\mathcal{C}, \mathcal{S}]^{(n)}$.

Proof. By Theorem 3.4 .2 the cogap map

$$
\lfloor h, k\rfloor: G \sqcup_{F} H \rightarrow K
$$

is a $P_{2 n+1}$-equivalence. Now note that $F$, as a limit of $(2 n+1)$-excisive functors, is $(2 n+1)$-excisive. Hence $P_{2 n+1}\left(G \sqcup_{F} H\right)$ is the pushout in the category $[\mathcal{C}, \mathcal{S}]^{(n)}$. So the cogap map in $[\mathcal{C}, \mathcal{S}]^{(n)}$

$$
P_{2 n+1}\left(G \sqcup_{F} H\right) \rightarrow K
$$

is a $P_{2 n+1}$-equivalence between $(2 n+1)$-excisive functors. Hence it is an isomorphism.

\section{A Truncated and connected maps of $n$-excisive functors}

Recall that every topos admits a factorization system consisting of the monomorphisms and covers (whose definition we will recall momentarily). The goal of this appendix is to describe this modality in the topos $[\mathcal{C}, \mathcal{S}]^{(n)}$ of $n$-excisive functors.

Definition A.0.1. A map $f: X \rightarrow Y$ in a topos $\mathcal{E}$ a monomorphism if its diagonal map

$$
\Delta f: X \rightarrow X \times_{Y} X
$$

is an isomorphism. A map is a cover if it is left orthogonal to every monomorphism. 
Example A.0.2. In the category of spaces $\mathcal{S}$, a map is a monomorphism if and only if it is the inclusion of a union of path components. The covers in spaces are exactly the maps that induce a surjection on the set of path components.

Before stating the next result, recall that an object $X$ in a category $\mathcal{C}$ is called discrete if the space $\mathfrak{C}(K, X)$ is discrete for every object $K \in \mathcal{C}$. Moreover, Lur09, Proposition 5.5.6.18] shows that if the category $\mathcal{C}$ is presentable (for example, if $\mathcal{C}$ is a topos) then the inclusion of the full subcategory $\mathcal{E}_{0} \rightarrow \mathcal{E}$ of discrete objects admits a left adjoint $\tau_{0}: \mathcal{E} \rightarrow \mathcal{E}_{0}$ which we will refer to as 0 truncation functor. A posteriori, we may characterize the discrete objects $X \in \mathcal{E}$ as those for which the canonical map $X \rightarrow \tau_{0} X$ is an isomorphism.

Example A.0.3. Here are examples of discrete objects.

1. If $\mathcal{E}=\mathcal{S}$, then $\tau_{0}$ is the functor sending a space $X$ to its set $\pi_{0} X$ of path components regarded as a discrete space.

2. If $\mathcal{E}=[\mathcal{C}, \mathcal{S}]$ it is not hard to see that

$$
\left(\tau_{0} F\right)(K)=\pi_{0}(F(K))
$$

for every $F \in \mathcal{E}$ and every $K \in \mathcal{C}$, so that a functor $F$ is discrete if and only if it takes values in discrete spaces.

The following folklore proposition asserts that monomorphisms and covers in a topos $\mathcal{E}$ are essentially determined by their restriction to discrete objects.

Proposition A.0.4. Let $f: X \rightarrow Y$ be a morphism in a topos.

(1) The map $f$ is a monomorphism if and only if $\tau_{0} f$ is a monomorphism and the square

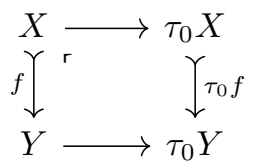

is a pullback.

(2) The map $f$ is a cover if and only if $\tau_{0} f$ is a cover.

We will prove the following result which characterizes monomorphisms and covers in the topos of $n$-excisive functors:

Theorem A.0.5. Let $f: F \rightarrow G$ be a map in $[\mathcal{C}, \mathcal{S}]^{(n)}$. Then:

(1) The map $f$ is monomorphism if and only if $P_{0} f$ is a monomorphism and the square

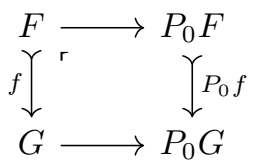

is a pullback. 
(2) The map $f$ is a cover if and only if $P_{0} f$ is a cover.

Remark A.0.6. We invite the reader to observe the similarity between Proposition A.0.4 and Theorem A.0.5. The category $[\mathcal{C}, \mathcal{S}]^{(0)}$ is equivalent to the category of spaces and the functor $P_{0}$ is equivalent to the evaluation functor $F \mapsto F(1)$. It follows from the theorem that a map $f: X \rightarrow Y$ in $[\mathcal{C}, \mathcal{S}]^{(n)}$ is a cover if and only if the map $f(1): X(1) \rightarrow Y(1)$ is a cover in the category of spaces.

We begin with some generalities: let us suppose that we have a left exact localization $P: \mathcal{E} \rightarrow \mathcal{F}$ with fully faithful right adjoint $i: \mathcal{F} \hookrightarrow \mathcal{E}$. We will write $\tau_{0}^{\mathcal{E}}$ and $\tau_{0}^{\mathcal{F}}$ for the 0 -truncation functors of $\mathcal{E}$ and $\mathcal{F}$ respectively. Now, it follows from the fact that $P$ preserves colimits that $P$ also commutes with 0 -truncation. That is

$$
P \tau_{0}^{\mathcal{E}} \simeq \tau_{0}^{\mathcal{F}} P
$$

On the other hand, the inclusion $i$ does not, in general, preserve colimits and hence when we identify $\mathcal{F}$ with a full subcategory of $\mathcal{E}$, we must distinguish between these two distinct operations.

Specializing to the case at hand, the following notation will be convenient:

Definition A.0.7. In what follows, we write $\tau_{0}$ for 0 -truncation in $[\mathcal{C}, \mathcal{S}]$ and $\tau_{0}^{(n)}$ for the 0 -truncation functor in the $n$-excisive localization $[\mathcal{C}, \mathcal{S}]^{(n)}$.

Remark A.0.8. The case $n=0$ here merits special attention. In this case, the functor $i:[\mathcal{C}, \mathcal{S}]^{(0)} \hookrightarrow[\mathcal{C}, \mathcal{S}]$ admits both a left and right adjoint given respectively by left and right Kan extension along the inclusion of the terminal object $1 \hookrightarrow \mathcal{C}$. As a consequence, the 0 -truncation functors $\tau_{0}^{(0)}$ and $\tau_{0} d o$ coincide on the essential image of $i$, which can be identified with the constant functors.

Lemma A.0.9. A discrete functor $F: \mathcal{C} \rightarrow \mathcal{S}$ is 1-excisive if and only it is constant.

Proof. If $F: \mathcal{C} \rightarrow \mathcal{S}$ is 1-excisive, then the following square is cartesian for every $K \in \mathcal{C}:$

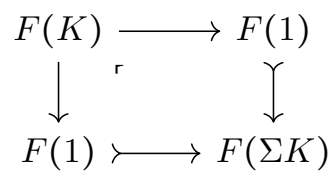

But the map $F(\Sigma K) \rightarrow F(1)$ is a left inverse of the map $F(1) \rightarrow F(\Sigma K)$, since the map $\Sigma K \rightarrow 1$ is a left inverse of the map $1 \rightarrow \Sigma K$. Hence the map $F(1) \rightarrow F(\Sigma K)$ is monic, since $F(\Sigma K)$ is discrete by hypothesis. It follows that the map $F(K) \rightarrow F(1)$ is invertible since the square above is cartesian.

The preceding lemma allows us to calculate the action of the 0-truncation functor $\tau_{0}^{(1)}$ in the category of 1-excisive functors. The result asserts that the 0 -truncation of a 1 -excisive $F$ is constant with value the 0 -truncation of the space $F(1)$. 
Lemma A.0.10. For $F \in[\mathcal{C}, \mathcal{S}]^{(1)}$, we have

$$
\tau_{0}^{(1)} F=\tau_{0} P_{0} F
$$

Proof. Note that the functor $\tau_{0}^{(1)} F$ is both discrete and 1-excisive by definition. According to Lemma A.0.9, then, it is constant. The functor $\tau_{0} P_{0} F$ is also constant and hence, to show that the two agree, it suffices to show they agree after evaluation at $1 \in \mathcal{C}$. But we have

$$
\tau_{0}^{(1)}(F)(1)=P_{0} \tau_{0}^{(1)}(F)(1)=\tau_{0}^{(0)} P_{0}(F)(1)=\tau_{0} P_{0}(F)(1)
$$

Where the first equality is by the definition of $P_{0}$, the second is an application of 5 to the localization $P_{0}:[\mathcal{C}, \mathcal{S}]^{(1)} \rightarrow[\mathcal{C}, \mathcal{S}]^{(0)}$, and the last follows from Remark A.0.8.

Proposition A.0.11. Every n-excisive monomorphism is 0-excisive.

Proof. The proof is by induction on $n \geq 0$. The case $n=0$ is obvious. Let us suppose $n=1$. So let $f: F \rightarrow G$ be a 1-excisive monomorphism. The map $P_{1} f$ is monic, since the functor $P_{1}$ is a left exact localization. The following square is cartesian, since $f$ is 1 -excisive.

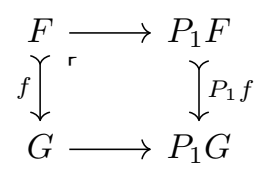

Hence it suffices to show that $P_{1} f$ is 0 -excisive. Hence we may suppose, without loss of generality, that $F$ and $G$ are in fact 1-excisive functors. Consider the cube:

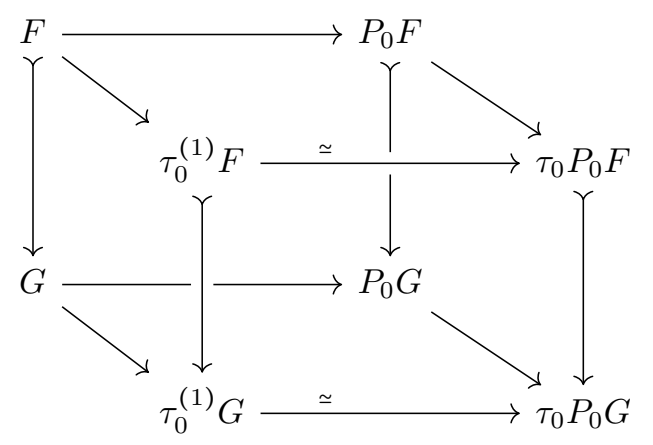

Let us show that the back face is a pullback. All of the vertical maps are monomorphisms since the functors $P_{0}, \tau_{0}$ and $\tau_{0}^{(1)}$ preserve them. Both the left and the right face are pullbacks by Proposition A.0.4. By Lemma A.0.10, the front two horizontal maps are in fact isomorphisms, since $F$ and $G$ are 1-excisive. Consequently, the back face is a pullback, which says that $f$ is 0 -excisive. 
For the inductive step, let $f: F \rightarrow G$ be a $(n+1)$-excisive monomorphism. Note that the functor $\left\{w_{K},-\right\}$ preserves monomorphisms. So, for all $K,\left\{w_{K}, f\right\}$ is a monomorphism. But it is also $n$-excisive by Theorem 3.3.4.(1). By the induction hypothesis, it is then 0 -excisive. This, in turn, shows that $f$ is 1 excisive. Then the case $n=1$ implies that $f$ is also 0 -excisive.

Proof of Theorem A.0.5. $(1 \Rightarrow)$. This is immediate since $P_{0}$ preserves monomorphisms and the pullback expresses just the statement that $f$ is 0 -excisive.

$(1 \Leftarrow)$. Monomorphisms are always stable by pullback.

$(2 \Rightarrow)$. The functor $P_{0}$ preserves covers because it is a localization.

$(2 \Leftarrow)$. Note that $f$ is a cover if and only if it is orthogonal to every monomorphism in $[\mathcal{C}, \mathcal{S}]^{(n)}$. So let $g: H \rightarrow K$ be such a monomorphism and consider a lifting problem as follows:

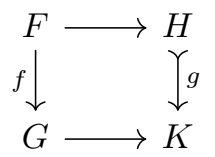

Note that since $g$ is a monomorphism, it is enough to show that a lift exists, as its uniqueness is automatic. Now apply the functor $P_{0}$ to obtain

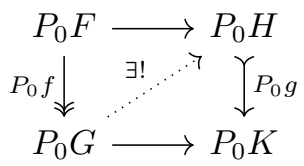

Observe that the left map is a cover by assumption. Since $P_{0}$ preserves monomorphisms, this square has a unique lift. But now composition of our lift with the map $p_{0} G: G \rightarrow P_{0} G$ yields the lift shown in the diagram:

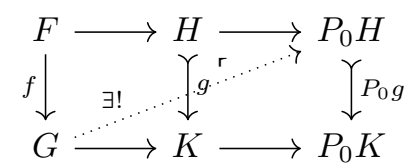

On the other hand, Proposition A.0.11 asserts that the right hand square is a pullback. Hence we have an induced unique lift to the original problem. This shows that $f$ is a cover.

\section{References}

[ABFJ17] Mathieu Anel, Georg Biedermann, Eric Finster, and André Joyal. A generalized Blakers-Massey theorem. https://arxiv.org/abs/1703.09050, 2017.

[ADL08] Gregory Z. Arone, William G. Dwyer, and Kathryn Lesh. Loop structures in Taylor towers. Algebr. Geom. Topol., 8(1):173-210, 2008. 
[BJM15] Kristine Bauer, Brenda Johnson, and Randy McCarthy. Cross effects and calculus in an unbased setting. Transactions of the American Mathematical Society, 367(9):6671-6718, 2015.

[BR14] Georg Biedermann and Oliver Röndigs. Calculus of functors and model categories, II. Algebr. Geom. Topol., 14(5):2853-2913, 2014.

[Goo90] Thomas G. Goodwillie. Calculus I: The First Derivative of Pseudoisotropy Theory. K-Theory, 4, 1990.

[Goo92] Thomas G. Goodwillie. Calculus II: Analytic Functors. K-Theory, 5, 1992.

[Goo03] Thomas G. Goodwillie. Calculus III: Taylor Series. Geometry and Topology, 7, October 2003.

[Heu15] Gijs Heuts. Goodwillie approximations to higher categories. arXiv:1510.03304, October 2015.

[Joy08] Andre Joyal. Notes on quasicategories. http://www.math.uchicago.edu/ may/IMA/Joyal.pdf, 2008.

[Kuh07] Nicholas J Kuhn. Goodwillie towers and chromatic homotopy: an overview. Proceedings of the Nishida Fest (Kinosaki 2003), 10:245$279,2007$.

[Lur09] Jacob Lurie. Higher topos theory, volume 170 of Annals of Mathematics Studies. Princeton University Press, Princeton, NJ, 2009.

[Lur16] Jacob Lurie. Higher algebra.http://www.math.harvard.edu/ lurie/, 2016.

[Rez05] Charles Rezk. Toposes and homotopy toposes. http://www.math.uiuc.edu/ rezk, 2005.

[Rez13] Charles Rezk. A streamlined proof of Goodwillie's n-excisive approximation. Algebr. Geom. Topol., 13(2):1049-1051, 2013.

[Wei95] Michael Weiss. Orthogonal calculus. Transactions of the American mathematical society, 347(10):3743-3796, 1995. 